\title{
1.14 Three-membered Rings with Two Oxygen and/or Sulfur Atoms
}

Mlostoń, Grzegorz ; Romanski, Jaroslaw ; Heimgartner, Heinz

\begin{abstract}
Combination of matrix isolation techniques with theoretical methods for identification and characterization of some dithiiranes and oxathiiranes is presented. In general, both dithiiranes and oxathiiranes were generated via flask vacuum pyrolysis of suitable sulfur-containing precursors such as 1,2,4-trithiolanes, 1,3dithietane 1,3-dioxide or thiocarbonyl S-oxides (sulfines). Novel applications of dimethyldioxirane (DMDO) and (trifluoromethyl)methyl dioxirane (TFMDO) as versatile oxidizing reagents published in the period 2008-2018 are summarized. Methods applied for manufacturing of new polymeric materials functionalized with dioxirane moieties and reported in patents are presented. Mechanisms involving Criegee intermediates in reactions of carbonyl compounds with dioxiranes using experimental and theoretical methods are discussed in the light of recent results.
\end{abstract}

DOI: https://doi.org/10.1016/B978-0-12-409547-2.14765-1

Posted at the Zurich Open Repository and Archive, University of Zurich

ZORA URL: https://doi.org/10.5167/uzh-185522

Book Section

Accepted Version

Originally published at:

Mlostoń, Grzegorz; Romanski, Jaroslaw; Heimgartner, Heinz (2022). 1.14 Three-membered Rings with Two Oxygen and/or Sulfur Atoms. In: Black, David. Comprehensive Heterocyclic Chemistry IV. Waltham, MA: Elsevier, 641-677.

DOI: https://doi.org/10.1016/B978-0-12-409547-2.14765-1 


\subsection{Three-membered Rings with Two Oxygen and/or Sulfur Atoms}

Grzegorz Mlostoń, ${ }^{a}$ Jarosław Romański ${ }^{a}$ and Heinz Heimgartner ${ }^{\mathrm{b}}$

${ }^{\text {a }}$ Department of Organic and Applied Chemistry, University of Łódź, Tamka Str. 12, PL-91-403 tódź, Poland,

E-mail: grzegorz.mloston@chemia.uni.lodz.pl; jaroslaw.romanski@chemia.uni.lodz.pl

${ }^{b}$ Department of Chemistry, University of Zurich, Winterthurerstrasse 190, CH-8057 Zurich, Switzerland,

E-mail: heinz.heimgartner@chem.uzh.ch

\section{Keywords:}

three-membered heterocycles, dithiiranes, oxathiiranes, dioxiranes, matrix isolation technique, spectroscopic methods, theoretical methods.

Abstract: Combination of matrix isolation techniques with theoretical methods for identification and characterization of some dithiiranes and oxathiiranes is presented. In general, both dithiiranes and oxathiiranes were generated via flask vacuum pyrolysis of suitable sulfur-containing precursors such as 1,2,4-trithiolanes, 1,3-dithietane 1,3-dioxide or thiocarbonyl S-oxides (sulfines). Novel applications of dimethyldioxirane (DMDO) and (trifluoromethyl)methyl dioxirane (TFMDO) as versatile oxidizing reagents published in the period 2008-2018 are summarized. Methods applied for manufacturing of new polymeric materials functionalized with dioxirane moieties and reported in patents are presented. Mechanisms involving Criegee intermediates in reactions of carbonyl compounds with dioxiranes using experimental and theoretical methods are discussed in the light of recent results. 


\subsubsection{Introduction}

Since the appearance of CHEC III (2008) ${ }^{(1)}$ research related to the chemistry of dithiiranes 1, oxathiiranes $\mathbf{2}$ and dioxiranes $\mathbf{3}$ has been presented in a series of books, review articles, and original publications. In analogy to the previous decade, the largest number of reports is related to the chemistry and especially new applications of disubstitued dioxiranes of type 3 . The parent dioxirane $3 a\left(R^{1}=R^{2}=H\right)$ has rarely been studied in the reviewed period. In contrast, dimethyldioxirane $\mathbf{3 b}\left(\mathrm{R}^{1}=\mathrm{R}^{2}=\mathrm{Me}\right)$ followed by the bis(trifluoromethyl) analogue $\mathbf{3 c}$ $\left(R^{1}=R^{2}=C F_{3}\right)$ were most frequently involved in the studies.

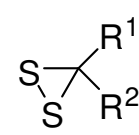

1

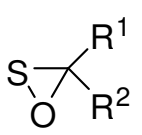

2

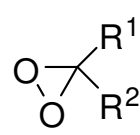

3

Figure 1

Whereas dithiiranes 1 and oxathiiranes $\mathbf{2}$ were subjected mainly to computational and spectroscopic studies in matrices, dioxiranes $\mathbf{3}$ found broad application as isolable or in situ generated compounds in diverse oxidation protocols. In this field of organic synthesis, asymmetric oxidations performed in the presence of a chiral catalyst leading to optically active oxiranes are of special interest and numerous examples were described in a comprehensive review. ${ }^{(2)}$ Another important application of dioxiranes 3 comprises the oxidations of heteroatoms $(\mathrm{N}, \mathrm{S}, \mathrm{Se}, \mathrm{I})$ and metal complexes, e.g. $\mathrm{Me}_{2} \mathrm{PtR}_{2}$, and they are summarized in a recent review. ${ }^{(3)}$ An extensive summary of the chemistry of dioxiranes $\mathbf{3}$, prepared by the pioneers of this class of compounds, was also published. ${ }^{(4)}$

Isolable oxathiiranes $\mathbf{2}$ are not known, but the parent representative $2 a\left(R^{1}=R^{2}=H\right)$ was generated under flash-vacuum pyrolysis conditions and identified for the first time after immobilization in Ar-matrix at $10 \mathrm{~K} .{ }^{(5)}$ Some spiro-oxathiiranes were characterized under the same conditions as products of thermal isomerization of cycloaliphatic thioketone S-oxides (sulfines) in the gas phase. ${ }^{(6)}$ In addition, their appearance was postulated to explain the socalled 'sulfur-transfer reaction' performed with 2 -unsubstituted imidazole $N$-oxides ${ }^{(7)}$ and some cycloaliphatic thioketones. ${ }^{(8)}$ In general, oxathiiranes $\mathbf{2}$ can be considered as isomeric cyclic forms of the corresponding thione S-oxides, and for that reason play an important role 
in the mechanisms of desulfurizations leading to carbonyl analogues. ${ }^{(9)}$ This mechanism of the sulfur-to-oxygen exchange is accepted for many reactions performed with thiocarbonyl $S$-oxides and numerous examples of such reactions are discussed in a recent review. ${ }^{(10)}$

In the period 2008-2018 no stable dithiirane derivatives were reported. Nevertheless, the earlier matrix isolation of the parent thiirane $1 a\left(R^{1}=R^{2}=H\right)^{(11)}$ was successfully repeated and its structure was studied using advanced computational methods. ${ }^{(12)}$ Some disubstituted dithiiranes are postulated as reactive intermediates formed after $(3+2)$-cycloelimination of 1,2,4-trithiolanes, and their trapping with $\mathrm{Pt}^{\circ}$-complexes leads to the corresponding dithiolato-Pt-complexes. ${ }^{(13)}$ Matrix isolation studies on small-ring sulfur heterocycles including dithiiranes as well as oxathiiranes have been summarized in a review. ${ }^{(14)}$

\subsubsection{Theoretical Methods}

It is generally known that the heterocycles 1-3 are unstable compounds and rarely could be isolated under special conditions, e.g. high dilution or immobilization in low-temperature matrices. For that reason, the structural studies of the derivatives or mechanisms of their reactions preferentially are performed using computational methods.

\subsubsection{Dithiiranes}

The parent dithiirane $\mathbf{1 a}$ and its precursor $\mathbf{5 a}$, generated in the gas phase from 1,2,4trithiolane $\mathbf{4 a}$, are believed to exist in equilibrium in the gas phase (Scheme 1). ${ }^{(11,12)}$

In solid matrix, the equilibrium was achieved with visible light $(\lambda=500 \mathrm{~nm})$. Irradiation at wavelength $>570 \mathrm{~nm}$ led to complete conversion of $\mathbf{5 a}$ into $1 \mathrm{1a}$. Relative enthalpies for both, 5 and 1a, were calculated based on the full CCSDTQ method and the energy barrier of the ring closure is predicted to be $27.0 \mathrm{kcal} \mathrm{mol}^{-1}$ (12) $^{(12)}$

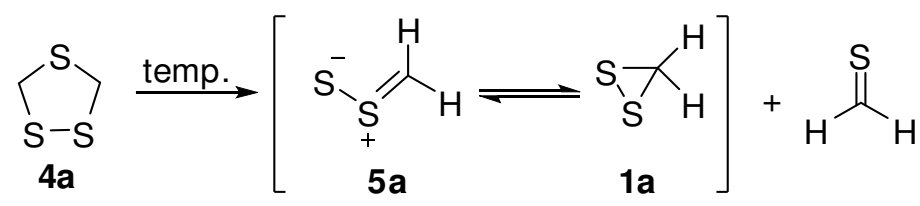

Scheme 1 
Second-order vibrational perturbation theory was applied to calculate harmonic and anharmonic vibrational frequencies, which offer an important tool for the identification of these elusive species in the gas phase. The calculations in the study, performed on the basis of $\operatorname{CCSD}(\mathrm{T})$ /aug-cc-pVTZ computation, led to the determination of bond distances and angles in $\mathbf{5 a}$ and $\mathbf{1 a}$ as well as in the transition state of their conversion (Figure 2). These studies can be considered as an extension of the long-lasting debate on the mechanism of ozonolysis reactions with the involvement of sulfur atoms (Criegee intermediates).

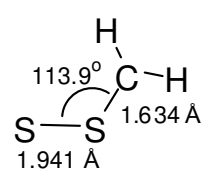

$5 a$

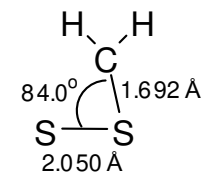

transition state

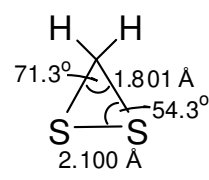

$1 \mathrm{a}$

Figure 2

Some spiroaliphatic thiosulfines and isomeric dithiiranes were also generated in the gas

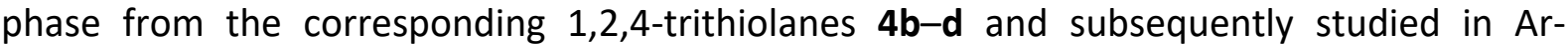
matrices at $10 \mathrm{~K}$ (Figure 3). ${ }^{(15)}$ The existence of both derivatives of type $\mathbf{5}$ and $\mathbf{1}$ was demonstrated by comparison of computed and registered IR and UV/Vis spectra. The calculation of the IR spectra was performed using the B3LYP/6-311+G(d,p) approach.

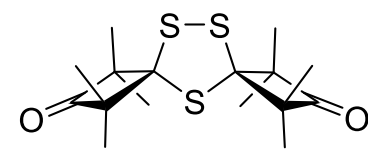

4b

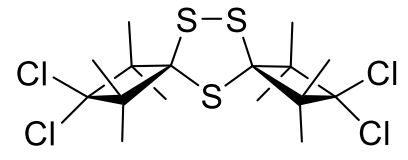

4c

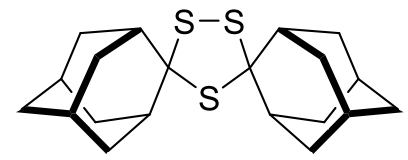

4d

Figure 3

The same method was applied for the detection of the presence of 5a and 1a in Ar-matrix after thermal (3+2)-cycloelimination reaction of the 1,2,4-trithiolane-4-oxide (6). The activation energy of this reaction, leading to the release of $5 \mathrm{a}$, was calculated to be $31.0 \mathrm{kcal}$ $\mathrm{mol}^{-1}$ (B3LYP/6-311+G(3df,3pd) level including zero-point vibrational energy corrections). ${ }^{(16)}$ The parent dithiirane 1a along with other cyclic and bicyclic disulfides was involved in the study aimed at the determination of excess electron binding in the gas phase. The 
compounds were investigated by ab initio MP2/6-31+G calculations. It has been shown that the ring size determines electron attachment, which is favored by the ring strain. ${ }^{(17)}$

The relative stability and optimized geometry of precursors for fullerene-like structures with the molecular formula $\mathrm{CS}_{2}$, which correspond to the cyclic structure 1a and the acyclic one 5a, were calculated based on the density functional theory (DFT). ${ }^{(18)}$

Computational studies were performed to explain the course of the reaction of $O\left({ }^{3} \mathrm{P}\right)$ with $\mathrm{CS}_{2}$. Among the possible structures with the molecular formula $\mathrm{COS}_{2}$, dithiiran-3-one (7) was also considered. ${ }^{(19)}$ The analogous molecule $\mathrm{CS}_{3}$ was found in the Ar-matrix at $4 \mathrm{~K}$ after cocondensation of carbondisulfide with discharged Ar. As the most likely structure of four possible isomers, dithiirane-2-thione (8) was found to be the most stable compound based on computations performed at the MP2 and B3LYP levels of theory. ${ }^{(20)}$
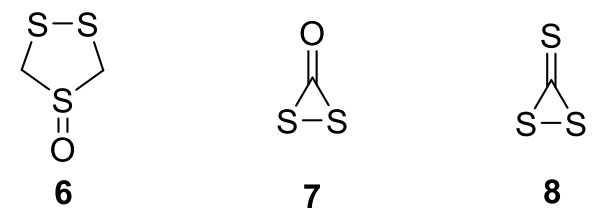

Figure 4

\subsubsection{Oxathiiranes}

The parent oxathiirane $2 a\left(R^{1}=R^{2}=H\right)$ was encountered for the first time in the mixture of products obtained after photochemical decomposition of thioformaldehyde S-oxide (10), generated by thermal (2+2)-cycloelimination of 1,3-dithiane-1,3-dioxide (9) in the gas phase (Scheme 2). ${ }^{(5)}$ The existence of $\mathbf{2 a}$ was demonstrated by comparison of the calculated and experimental IR spectra. The computation was carried out on the CCSD(T)/cc-pVTZ level of theory.

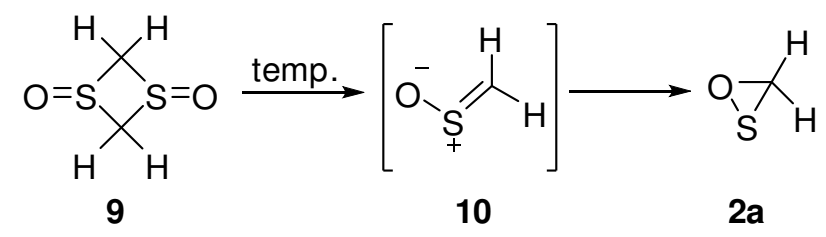

\section{Scheme 2}


The calculated bond lengths for $\mathbf{2 a}$ are depicted in the structure below (CCSD(T)/cc-pVTZ). The same method was used to establish the structural details of the isomeric compound 11, which was formed from 10 after irradiation with UV light (254 nm, Ar-matrix, $11 \mathrm{~K}) .^{(21)}$

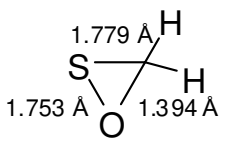

$2 a$

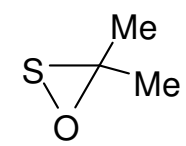

$2 b$<smiles></smiles>

11

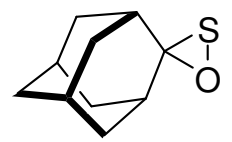

2c

Figure 5

The 2,2-disubstituted oxathiiranes $\mathbf{2} \mathbf{b}$ and $\mathbf{2} \mathbf{c}$ were also reported, and their identification comprised the comparison of experimental and computed UV and IR spectra (B3LYP/6$311+\mathrm{G}(3 \mathrm{df}, 3 \mathrm{pd})$ level). ${ }^{(6)}$

Desulfurization of $\mathbf{2} \mathbf{b}$ in the Ar-matrix was observed when the temperature rose up to $c a .40$ $\mathrm{K}$, and the progress of this reaction was monitored by IR spectroscopy. The formation of acetone and $\mathrm{S}_{2}$ was explained by the mechanism presented in Scheme $3 .{ }^{(6)}$ The computed energies of the products of this stepwise transformation showed that the process is highly exothermic $\left(\Delta \mathrm{H}=-62.8 \mathrm{kcal} \mathrm{mol}^{-1}\right)$. Analogous calculations were also performed for the parent $2 \mathrm{a}$ indicating a similar exothermicity $\left(\Delta \mathrm{H}=-51.4 \mathrm{kcal} \mathrm{mol}{ }^{-1}\right)$.

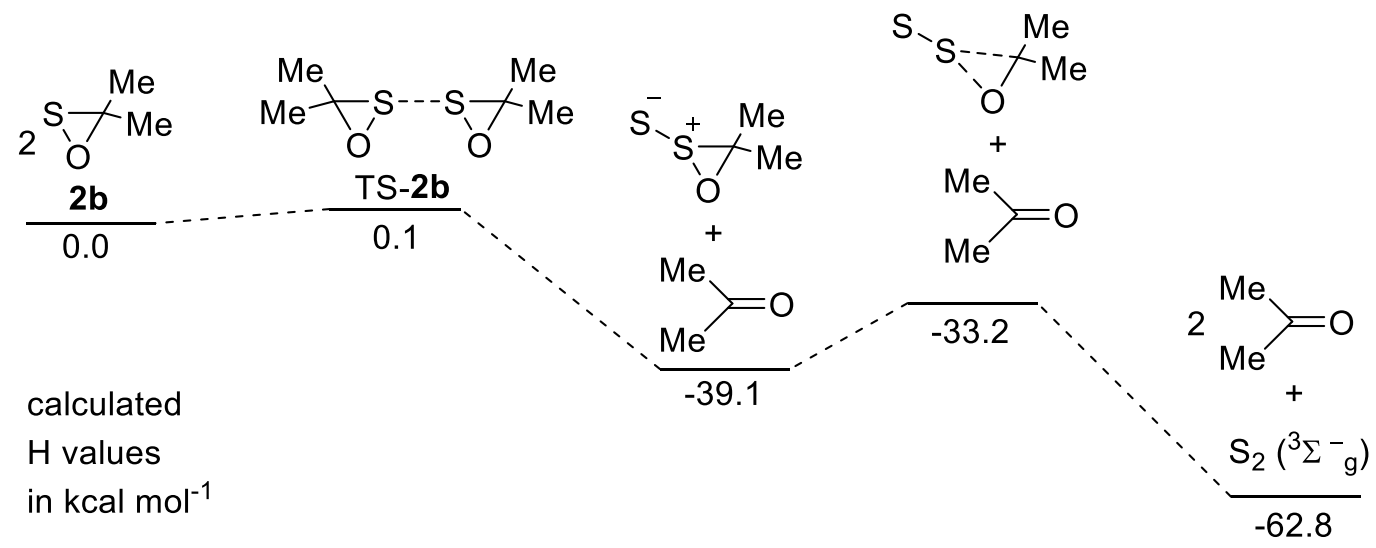

\section{Scheme 3}


An analogous reaction mechanism initiated by the $S \cdots \cdot S$ interaction was formulated and supported by computation for the parent thiirane. ${ }^{(22)}$

\subsubsection{Dioxiranes}

\subsection{General aspects}

Using computational methods, a full-dimensional potential energy surface was developed for dioxirane (3a). ${ }^{(23)}$ The applied method was based on an ab initio calculation at the CCSD(T)-F12a/AVTZ level. In addition, the IR absorptions were predicted. Vibrational energy levels for difluorodioxirane (3d) were computed using variational and perturbative methods. ${ }^{(24)}$ Both approaches gave comparable results, which were in good agreement with experimental values. The nature of the $0 \cdots 0$ interaction in protonated dioxirane-3-one was studied by using the natural bond orbital (NBO) method in conjunction with the localized molecular orbital energy decomposition analysis (LMOEDA). ${ }^{(25)}$ The computation showed that the $\mathrm{O} \cdots \mathrm{O}$ interaction exhibits the nature of a dative covalent bond.

\subsection{Epoxidation reactions}

The epoxidation of differently substituted alkenes using dimethyldioxirane (DMDO, 3b) was investigated by computational methods. ${ }^{(26)}$ For these reactions, transition states and activation barriers, both in acetone solution and in the gas phase, were calculated with the $B 3 L Y P / 6-311+G(d)$ level of theory. The study showed that the type of substituents of the alkene influences the epoxidation process. Whereas lower activation energies and early transition states are characteristic for electron-donating substituents, higher activation barriers and late transition states were obtained for electron-withdrawing groups. Chargetransfer (CT) from the alkene to the dioxirane reflects the electrophilic character of $\mathbf{3 b}$.

The epoxidation of para-benzoquinone-fused norbornadiene 12 by means of DMDO (3b) resulted in the formation of a 75:25 mixture of exo- and endo-epoxides 13 (Scheme 4). The observed stereoselectivity was rationalized by computing the reaction in the gas phase with the exchange-correlation functional B3LYP at $6-31+G(d, p)$ theory level. ${ }^{(27)}$ The solvent effect for dichloromethane was calculated at the same level using the CPCM solvation model. 


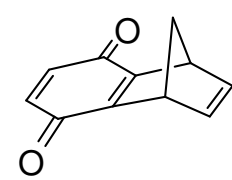

12
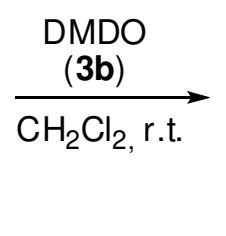

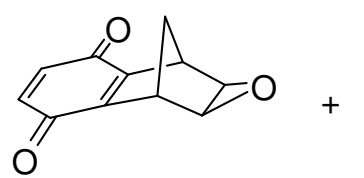

exo-13

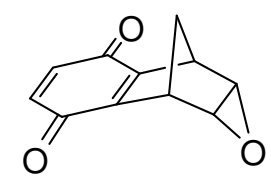

endo-13

\section{Scheme 4}

Similar epoxidations of 1,3-cyclohexadiene and 1,3-cyclooctadiene with DMDO (3b) were studied by computational modeling. ${ }^{(28)}$ For both compounds, basic density functional calculations at the B3LYP/6-31G level were applied to model the mono-oxidation processes. The mechanism formulated for the formation of the epoxide ring corresponded to a concerted electrophilic transfer of the $\mathrm{O}$-atom to the $\mathrm{C}=\mathrm{C}$ bond. The same mechanism was formulated in a similar study using the B3LYP DFT functional. ${ }^{(29)}$

\subsection{Insertion of the O-atom into the $\mathrm{C}-\mathrm{H}$ bond}

In contrast to epoxidations, insertion reactions leading to hydroxyl groups were found to proceed as non-concerted processes. This mechanism was already known and described in CHEC III (2008). ${ }^{(1)}$ A recent study aimed at reexamination of the stepwise pathway based on the application of DFT and G(4) molecular orbital calculations. In that case, the UM062X DFT functional led to the most accurate values of the $\mathrm{O}-\mathrm{O}$ bond dissociation energy. ${ }^{(29)}$ The initial $\mathrm{H}$ '⿳O口O interaction followed by the formation of an 'intimate radical pair' 14 and subsequent 'oxygen rebound' is the best representation of the computed pathway.<smiles>[R]C([R])[CH-]OC1(C)OO1</smiles>

3b

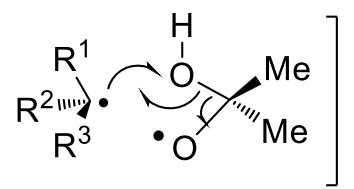

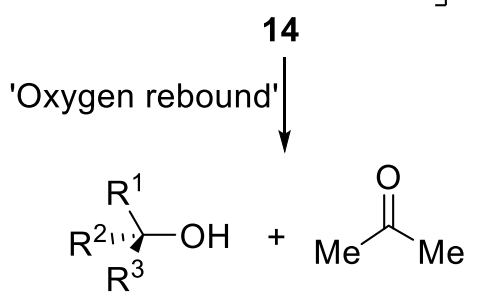

\section{Scheme 5}




\subsection{Dioxiranes as intermediates in ozonolysis reactions}

The mechanism of isoprene ozonolysis was studied based on quantum chemical and RRKM/master equation calculations. ${ }^{(30)}$ The intermediate acrolein O-oxide $\mathbf{1 5}$ is postulated to undergo 1,3-dipolar electrocyclization in an exothermic process (Scheme 6). The resulting dioxirane 16 decomposes via splitting of the $\mathrm{O}-\mathrm{O}$ bond generating an unstable diradical 17, followed by elimination of atomic oxygen to give acrolein as final product.

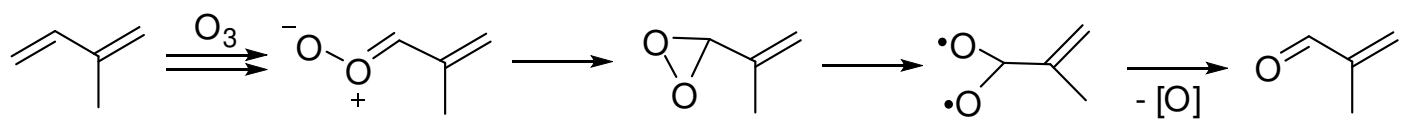

15

16

17

\section{Scheme 6}

Computational methods were also applied to elucidate reaction mechanisms which determine the type of products of the ozonolysis of $\alpha$-phellandrene. ${ }^{(31)}$ Also in this study, dioxiranes were postulated as intermediates formed from the previously generated carbonyl O-oxides after cleavage of the primary ozonides.

\subsubsection{Experimental Structural Methods}

In the reviewed period, these methods were limited to UV/Vis and IR spectra registered for some derivatives of dithiiranes and oxathiiranes immobilized in Ar-matrices. No X-ray crystal structures were reported for all three classes of compounds 1-3.

\subsubsection{Dithiiranes}

\subsection{Spectroscopic studies}

The spirocyclic dithiirane $\mathbf{1 b}$ was identified in the pyrolysate obtained after decomposition of 1,2,4-trithiolane $\mathbf{4 b}$ in the gas phase at $600{ }^{\circ} \mathrm{C}$ by IR spectroscopy in Ar-matrix at $10 \mathrm{~K}$ (Figure $6) .{ }^{(15)}$ The registered absorptions fitted well with those of the calculated values. The most characteristic absorption bands were found at ca. 1780, 1450 and $1030 \mathrm{~cm}^{-1}$. Similarly, 
spirodithiirane 1c was characterized in the Ar-matrix at $10 \mathrm{~K}$, and in this case, the most intense band appears at $1450 \mathrm{~cm}^{-1}$.

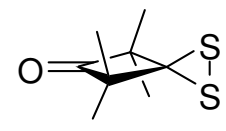

$1 \mathrm{~b}$

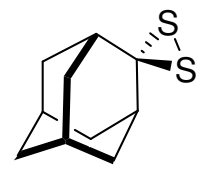

$1 \mathrm{c}$

Figure 6

\subsubsection{Oxathiiranes}

The first experimental IR spectrum of the parent oxathiirane (2a) was reported by Schreiner et al. ${ }^{(5)}$ The spectrum was measured in Ar-matrix at $11 \mathrm{~K}$ for a mixture of products after photolysis of thioformaldehyde S-oxide (10), and the absorption bands found in the IR difference spectrum corresponded well with the calculated values. The most characteristic absorptions are located at ca. 690 and $1130 \mathrm{~cm}^{-1}$. In the case of 3,3-dimethyloxathiirane (2b), the experimental IR spectrum was also described for the product immobilized in Armatrix after photolysis of thioacetone $S$-oxide. ${ }^{(6)}$ Three most intense absorptions were localized at 807,1096 , and $1267 \mathrm{~cm}^{-1}$; they are also in good agreement with the calculated ones. In the third example of an experimental IR spectrum of spiro[adamantane-2,3'oxathiirane] (2c), the most intense absorptions appeared at 821, 1146, and $1452 \mathrm{~cm}^{-1}$ and they correlated with the computed frequencies. ${ }^{(6)}$ The experimental UV/Vis spectrum of $2 \mathrm{c}$ revealed an intense absorption maximum at $\lambda=216.6 \mathrm{~nm}$ and a second very weak one at $\lambda=$ $424.6 \mathrm{~nm}$.

\subsubsection{Dioxiranes}

No new experimental spectroscopic data are available for dioxiranes in the reviewed period. 


\subsubsection{Thermodynamic Aspects}

No isolable representatives stable at ambient conditions were reported between 2008 and 2018.

1.14.5 Reactivity of Three-Membered Rings with Two Oxygen and/or Sulfur Atoms

\subsubsection{Dithiiranes}

Spirocycloaliphatic dithiiranes $\mathbf{1 b} \mathbf{b} \mathbf{d}$ were obtained in Ar-matrix at $10 \mathrm{~K}$ after photolysis of the corresponding thiocarbonyl S-sulfides (thiosulfines) (Scheme 7). ${ }^{(15)}$ In the cases of sterically congested dithiiranes $\mathbf{1} \mathbf{b}$ and $\mathbf{1} \mathbf{d}$, further irradiation led to their isomerization to give dithiolactones $\mathbf{1 8 b}$ and $\mathbf{1 8 d}$, respectively. Both compounds were identified by IR spectroscopy. In contrast, the attempted photoisomerization of 1c was unsuccessful.

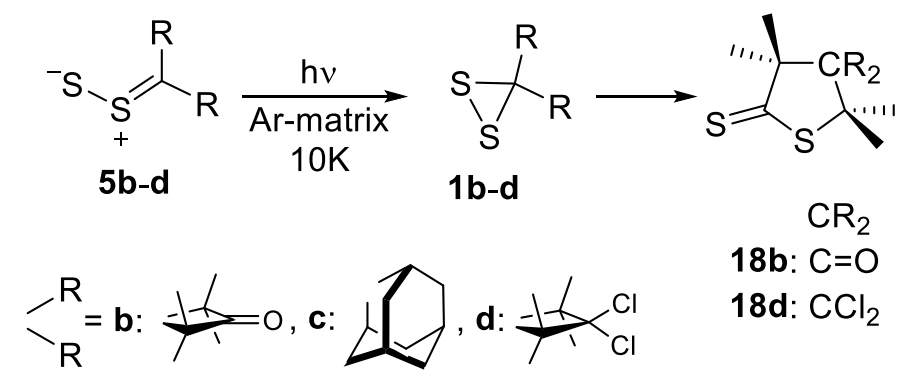

Scheme 7

The proposed explanation of the reaction mechanism is presented in Scheme 8: homolytic cleavage of the $S-S$ bond leads to the diradical 19, which undergoes opening of the cyclobutane ring yielding the delocalized diradical 20. This intermediate isomerises via ring closure to form dithiolactone $\mathbf{1 8 b}$. 


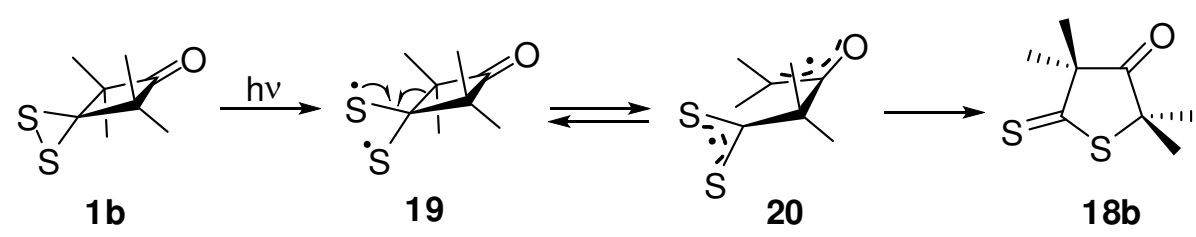

\section{Scheme 8}

Thermal (3+2)-cycloelimination of 3,3,5,5-tetraphenyl-1,2,4-trithiolane $4 \mathrm{e}$ in toluene solution leads to the formation of thiobenzophenone $S$-sulfide (5e), which exists in equilibrium with 3,3-diphenyldithiirane $1 e^{(13)}$ The latter is trapped by $\mathrm{Pt}^{\circ}$-complexes of type $\mathrm{P}_{2} \mathrm{Pt}\left(\eta^{2}\right.$-nbe) yielding dithiolato complexes 21 (Scheme 9). The eliminated thiobenzophenone is also trapped with the $\mathrm{Pt}^{\circ}$-complexes and so-called platina-thiiranes were formed in equimolar amounts.

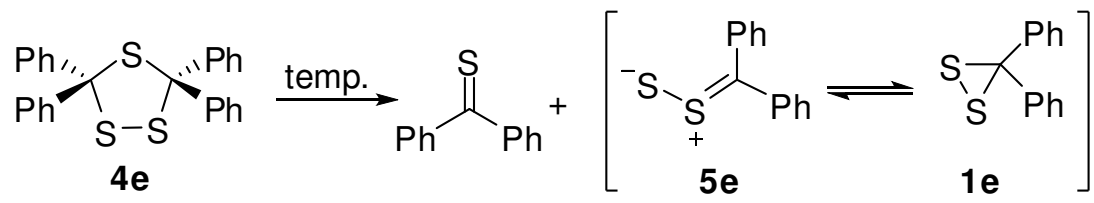

$$
\begin{aligned}
& C_{P}^{P}-P t\left(\eta^{2}-n b e\right) \mid-n b e \\
& \left(\mathrm{P}_{\mathrm{P}}-\mathrm{Pt}_{\mathrm{S}}^{-\mathrm{S}} \mathrm{Y}_{\mathrm{P}}^{\mathrm{Ph}}\right.
\end{aligned}
$$

21

\section{Scheme 9}

The parent 1,2,4-trithiolane $4 \mathrm{a}$ is common in nature, e.g., it was found in stinky bean seeds (Parkia speciosa), and it is believed to release $\mathrm{H}_{2} \mathrm{~S}$ formed in a multistep enzymatic reaction. The proposed mechanism of the $\mathrm{H}_{2} \mathrm{~S}$ formation is based on the assumption that $4 \mathrm{a}$ in the presence of glutathione is converted into methylene sulfide-conjugates and methylene dithiole, which is the precursor of $1 \mathbf{a} .^{(32)}$ Further conversion induced by glutathione leads to the formation of $\mathrm{H}_{2} \mathrm{~S}$.

\subsubsection{Oxathiiranes}

In analogy to dithiiranes $\mathbf{1 b}, \mathbf{d}$, photolytic isomerization of the matrix-isolated species $\mathbf{2} \mathbf{d}$ with $\lambda=313 \mathrm{~nm}$ light led to the formation of ring-enlarged products, i.e., thiolane-2,4-dione 22 
and 5-thioxotetrahydrofuran-3-one 23 (Scheme 10). ${ }^{(6)}$ In contrast to dithiirane 1c, the corresponding spiro[adamantane-2,3'-oxathiirane] (2c) undergoes the ring enlargement upon irradiation with $435 \mathrm{~nm}$ light, yielding only thiolactone 24.

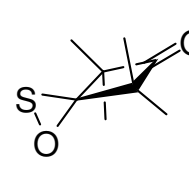

2d

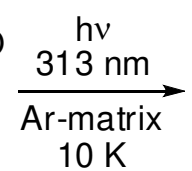

$10 \mathrm{~K}$

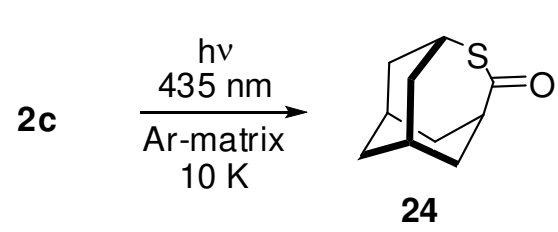

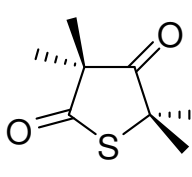

22

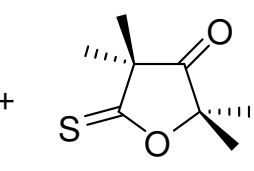

23

\section{Scheme 10}

All products were identified in matrices based on the IR spectra. It seems likely that in analogy to products $\mathbf{1 8}$, the formation of 22-24 occurs via intermediate diradicals, and in the case of $\mathbf{2 d}$, competitive ring closure of the intermediate diradical via $\mathrm{O}$ or $\mathrm{S}$ attack explains the formation of the mixture of the isomeric products $\mathbf{2 2}$ and $\mathbf{2 3 .}$

The reported formation of a mixture of isomeric products 11, 25-28 observed upon photolysis of thioformaldehyde S-oxide (10) can be explained by secondary photoreactions of the initially formed parent oxathiirane (2a) (Figure 7). ${ }^{(5)}$<smiles>O</smiles>

11<smiles></smiles>

25<smiles>OC=S</smiles>

26<smiles>O=CS</smiles>

27<smiles>OC=S</smiles>

28

Figure 7

Oxathiirane $\mathbf{2} \mathbf{d}$ was generated in situ as a product of 1,3-ring closure of the zwitterionic intermediate 29, formed from a sterically crowded 1-adamantylimidazole $\mathrm{N}$-oxide with 2,2,4,4-tetramethyl-3-thioxocyclobutanone acting as a dipolarophile. When the reaction was carried out in the presence of thiobenzophenone, the $S$-atom was transferred to the $C=S$ group generating thiobenzophenone $S$-sulfide $(\mathbf{5 e})$ as a reactive 1,3 -dipole (Scheme 11$){ }^{\left({ }^{(8)}\right.}$ 


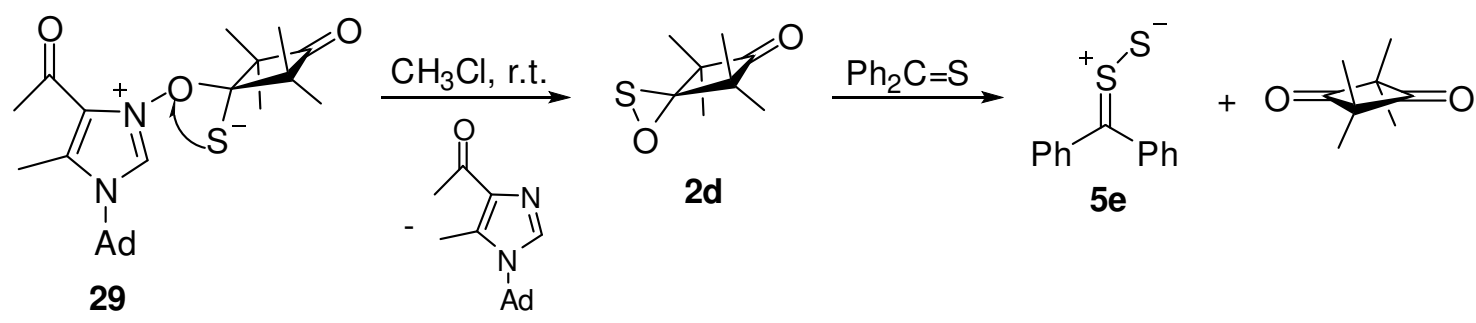

Scheme 11

The appearance of intermediate oxathiiranes was proposed in efficient conversions of thioamides, thioesters, and thioketones into the corresponding carbonyl derivatives. ${ }^{(9)}$ These reactions were performed using hydrogen peroxide $\left(\mathrm{H}_{2} \mathrm{O}_{2}\right)$ in the presence of an equimolar amount of trimethylsilyl chloride (TMSCl). The role of the latter was the activation of $\mathrm{H}_{2} \mathrm{O}_{2}$ and acceleration of the oxathiirane formation.

\subsubsection{Dioxiranes}

Dioxiranes are widely applied as versatile oxidizing reagents, which found applications both in academia and in large scale processes. ${ }^{(4)}$ The best known reactions are epoxidations of alkenes, hydroxylation of $\mathrm{C}-\mathrm{H}$ bonds, and oxidations of heteroatoms. The use of methyl(trifluoromethyl)dioxirane (3d) in all types of these oxidation reactions was summarized in a recent review. ${ }^{(33)}$

\subsection{Insertion of the O-atom into the $\mathrm{C}-\mathrm{H}$ bond}

The oxidation of cubane with methyl(trifluoromethyl)dioxirane (3d) in acetone or $\mathrm{CH}_{2} \mathrm{Cl}_{2}$ solution at $\mathrm{ca} .0^{\circ} \mathrm{C}$ led selectively to cubanol (30) in almost quantitative yield (Scheme 12). ${ }^{(34)}$ Notably, the next oxidation step afforded cubane-1,4-diol (31) in a selective manner and excellent yield.

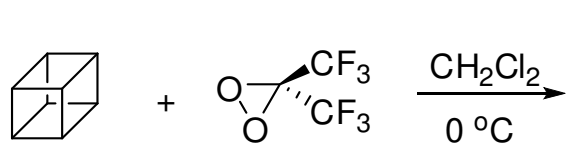

3d

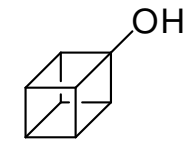

$30(98 \%)$

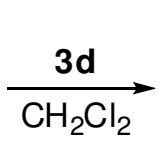

$0^{\circ} \mathrm{C}$

$\mathrm{HO}$

$31(95 \%)$

\section{Scheme 12}


The reaction was performed with isolated $\mathbf{3} \mathbf{d}$, which was used in a solution of trifluoropropanone (TFP). The rate of the insertion reaction for cubane was compared with those of adamantane, cubanol (30), and cyclopropane. The reaction rates showed that adamantane is the most reactive substrate followed by cubane. The mechanism proposed by the authors for the oxidation of cubane was a concerted but not synchronous one.

A similar study performed with homodiamantane (32) and dimethyldioxirane (3b) led to a mixture of apically and medially hydroxylated diamantanes $\mathbf{3 3 a}$ and $\mathbf{3 3 \mathbf { b }}$ (Scheme 13). ${ }^{\left({ }^{35)}\right.}$ The ratio of both products was shown to depend on the barriers of the oxygen insertion calculated with density functional theory methods.

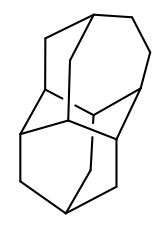

32

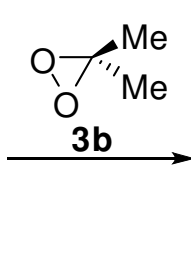

$3 b$

\section{Scheme 13}

In a recent publication, the first example of a ketone-catalyzed hydroxylation of a $\mathrm{C}-\mathrm{H}$ bond with aqueous $\mathrm{H}_{2} \mathrm{O}_{2}$ was reported. ${ }^{(36)}$ The reaction occurred not only chemo- but also stereoselectively and it took place only at tertiary $\mathrm{CH}$ groups (Scheme 14). The hydroxylation is postulated to proceed via an in situ generated 3-aryl-3-trifluoromethyldioxirane, and the best yield of hydroxylated product was obtained by using the 4-fluorophenyl derivative $\mathbf{3 e}$. The corresponding ketone, i.e. 4-fluorophenyl trifluoromethyl ketone, was used as a catalyst (0.2 mol equiv.).

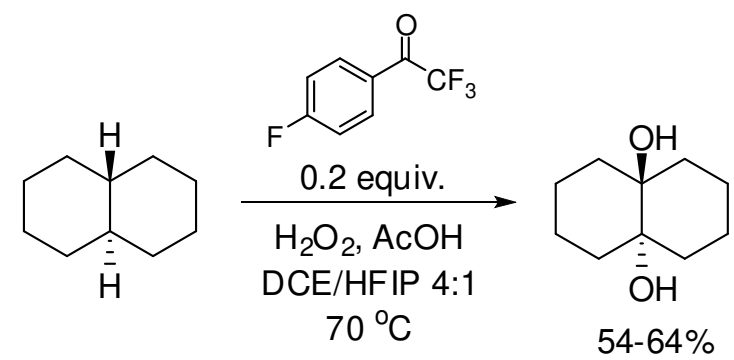

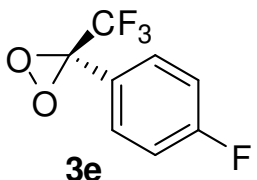

$3 e$

\section{Scheme 14}


Similar reactions with $\mathbf{3 e}$ as oxidizing agent were performed using the continuous flow platform, and under these conditions, not only tertiary alcohols but also benzylic alcohols were obtained in high yields. ${ }^{(37)}$

The hydroxylation reaction of isobutane with dimethyldioxirane (3b) leading to tert-butanol and acetone was studied computationally and short-lived diradical species were found in the 'oxygen rebound trajectories'. The time gap between $\mathrm{C}-\mathrm{H}$ bond breaking and $\mathrm{C}-\mathrm{O}$ bond formation was calculated to be between 30 and 150 femtoseconds. ${ }^{(38)}$

\subsection{Epoxidation reactions}

Epoxidation of diverse alkenes with dimethyldioxirane (3b) and methyl(trifluoromethyl)dioxirane (3e) demonstrated that irrespective of the nature of the alkene substituents, oxygen transfer to the $C=C$ bond occurs electrophilically, i.e., dioxirane acts as electrophile. ${ }^{(39)}$ Kinetic studies showed that in substituted cinnamonitriles the $p$ methoxy derivative reacts 20 times faster than the $m$-nitro analogue. In the same study, the oxidation of 1,1-diphenyl-2-vinylcyclopropane $\mathbf{3 4}$ with $\mathbf{3 b}$ leading to the corresponding oxirane $\mathbf{3 5}$ was formulated as a concerted process (Scheme 15). The intermediacy of diradicals was excluded. These results are in line with conclusions presented in a theoretical study. ${ }^{(26)}$

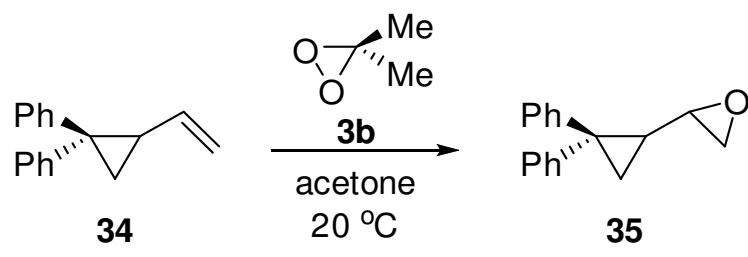

\section{Scheme 15}

Polydioxirane $\mathbf{3 6}$ was shown to be a new and powerful epoxidizing reagent for both alkyland aryl-substituted alkenes. ${ }^{(40)}$ In addition, the $\mathrm{CH}_{2} \mathrm{OH}$ group was tolerated by this reagent. Comparable results were obtained using prepared $\mathbf{3 6}$ or using it generated in situ. 


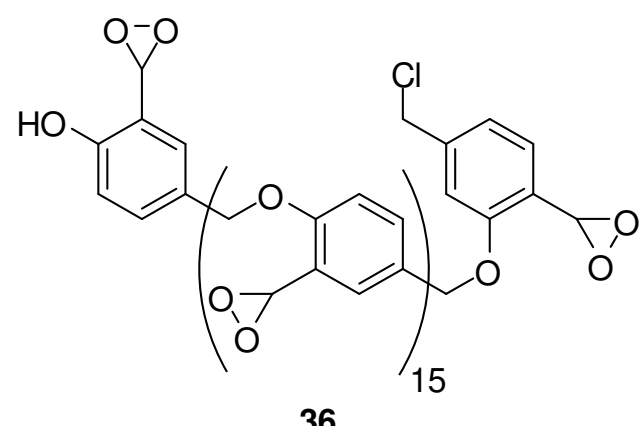

\section{Figure 8}

Cyclic conjugated dienes and trienes were extensively studied as substrates for epoxidation reactions by treatment with $\mathbf{3 b}$ and $\mathbf{3 d} .{ }^{(41)}$ For example, cyclopentadiene and cyclohexa-1,3diene gave the corresponding trans-bisepoxides. In the second case, the trans-isomer was formed as almost exclusive product in nearly quantitative yield. Epoxidations of cycloheptatriene lead to a mixture of diepoxide trans-37 and triepoxide trans,trans-38 in a ratio of ca. 9:1 (Scheme 16). The isolated trans-37 undergoes further epoxidation to yield the triepoxide trans,cis-38. Notably, dioxirane $\mathbf{3 d}$ reacted remarkably faster than $\mathbf{3 b}$, and the reaction times for completion of the reactions were determined to 10 and $180 \mathrm{~min}$, respectively.

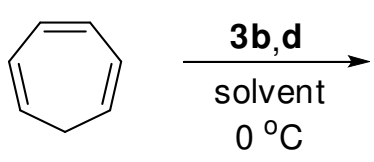

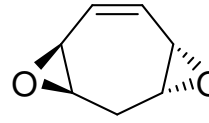

trans-37

$3 d \mid \begin{aligned} & \mathrm{CCl}_{4} \\ & 0^{\circ} \mathrm{C}\end{aligned}$

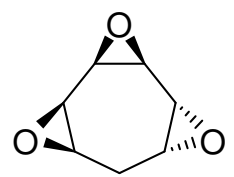

trans, cis-38

\section{Scheme 16}

Monoepoxidation of cyclohexa- and cyclohepta-1,3-dienes were achieved by using $\mathbf{3 b}$ in acetone solution at room temperature within several minutes, and according to GC/MS analysis, the products were obtained in excellent yields. ${ }^{(28)}$ 
Naturally occurring alkenes, e.g. terpenes, were extensively studied as substrates for both ozonolysis and epoxidation reactions. In the first case, dioxiranes are postulated as important intermediates in multistep processes. ${ }^{(31)}$ In a recent publication, an improved protocol for epoxidation of terpenes, such as $\beta$-pinene, carveol, carvone, and farnesol, using in situ-generated $\mathbf{3 b}$ under ultrasound irradiation was reported (Scheme 17). ${ }^{(42)}$ For example, the epoxidation of carvone (39) was complete after $6 \mathrm{~min}$, and the monoepoxide $\mathbf{4 0}$ was obtained quantitatively, but stereochemical aspects have not been considered.<smiles>C=C(C)C1CC=C(C)C(=O)C1</smiles>

39

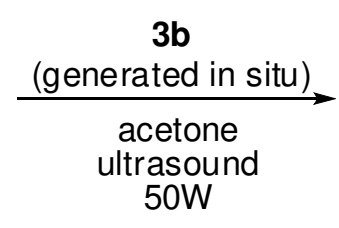

$50 \mathrm{~W}$

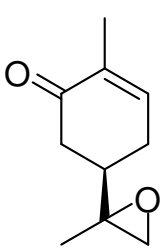

40

\section{Scheme 17}

Epoxidations using dioxiranes $\mathbf{3} \mathbf{b}$ and $\mathbf{3} \mathbf{d}$ found many applications in multistep procedures developed for the synthesis of natural products and conversions of sugar derivatives. Thus, some derivatives of isofagomine were prepared starting with the epoxide $\mathbf{4 1}$ obtained stereoselectively from the corresponding 3,4-dehydropiperidine derivative by treatment with $\mathbf{3 b}$ in $84 \%$ yield. ${ }^{(43)}$

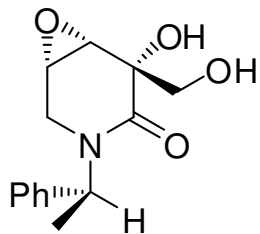

41

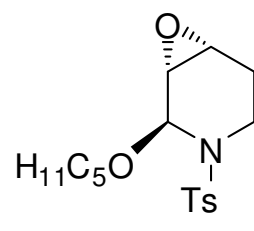

42

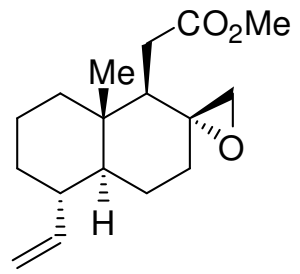

43

Figure 9

Oxidation of a $\mathrm{N}$-tosylated derivative of a 2-alkoxy-3,4-dehydropiperidine opens access to the epoxide 42 via treatment with excess $3 \mathbf{b}$ in acetone at $-78^{\circ} \mathrm{C}$ to room temperature. ${ }^{(44)}$ In this case, the diastereoselectivity was established to be 4:1. A similar $N$-tosylated 2,3dehydropiperidine derivative was oxidized using $\mathbf{3 b}$ in $\mathrm{CH}_{2} \mathrm{Cl}_{2}$, and the expected epoxide was 
obtained as a single diastereoisomer. ${ }^{(45)}$ This product was converted into the corresponding trans-configured 2-allylpiperidin-3-ol derivative in a regio-and stereoselective manner.

The total synthesis of $(+)$-asperolide $C$ includes the epoxide $4 \mathbf{4 3}$, which was prepared in a chemo- and diastereoselective manner from the corresponding methylidene derivative by using $\mathbf{3 b}$ in acetone at -78 to $-20^{\circ} \mathrm{C}^{(46)}$

The polycyclic epoxide $\mathbf{4 5}$ is an important intermediate for the synthesis of a chlorohydrine from (-)-shikimic acid. A convenient way for its preparation is offered by the highly selective epoxidation of the cyclic diene $\mathbf{4 4}$ with $\mathbf{3 d}$ (Scheme 18). ${ }^{(47)}$ In this case, the reaction at $-15^{\circ} \mathrm{C}$ afforded the desired epoxide $\mathbf{4 5}$ as the exclusive product. In contrast, similar oxidations with 3b or $m$-CPBA were less selective and led to mixtures of both isomers $\mathbf{4 5}$ and $\mathbf{4 6 .}$

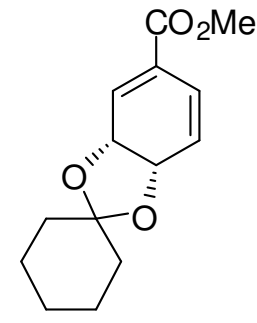

44

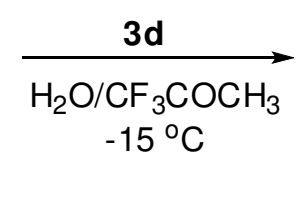

$-15^{\circ} \mathrm{C}$

\section{Scheme 18}

In a study aimed at the preparation of illicium sesquiterpene natural product analogues, the polycyclic epoxide $\mathbf{4 8}$ is an important building block, which is available via epoxidation of the cyclopentene fragment of the $\alpha$-oxocarboxylate 47 (Scheme 19). ${ }^{(48)}$ Interestingly, the attempted reaction of $\mathbf{4 7}$ with $\mathbf{3 d}$ led to a mixture of lactones $\mathbf{4 9}$ and $\mathbf{5 0}$, whereas the analogous reaction performed, after saponification of the ester group $\left(\mathrm{EtO}_{2} \mathrm{C}\right)$, with $\mathbf{3} \mathbf{b}$ gave the desired epoxide $\mathbf{4 8}$ in a stereoselective manner and in quantitative yield. 


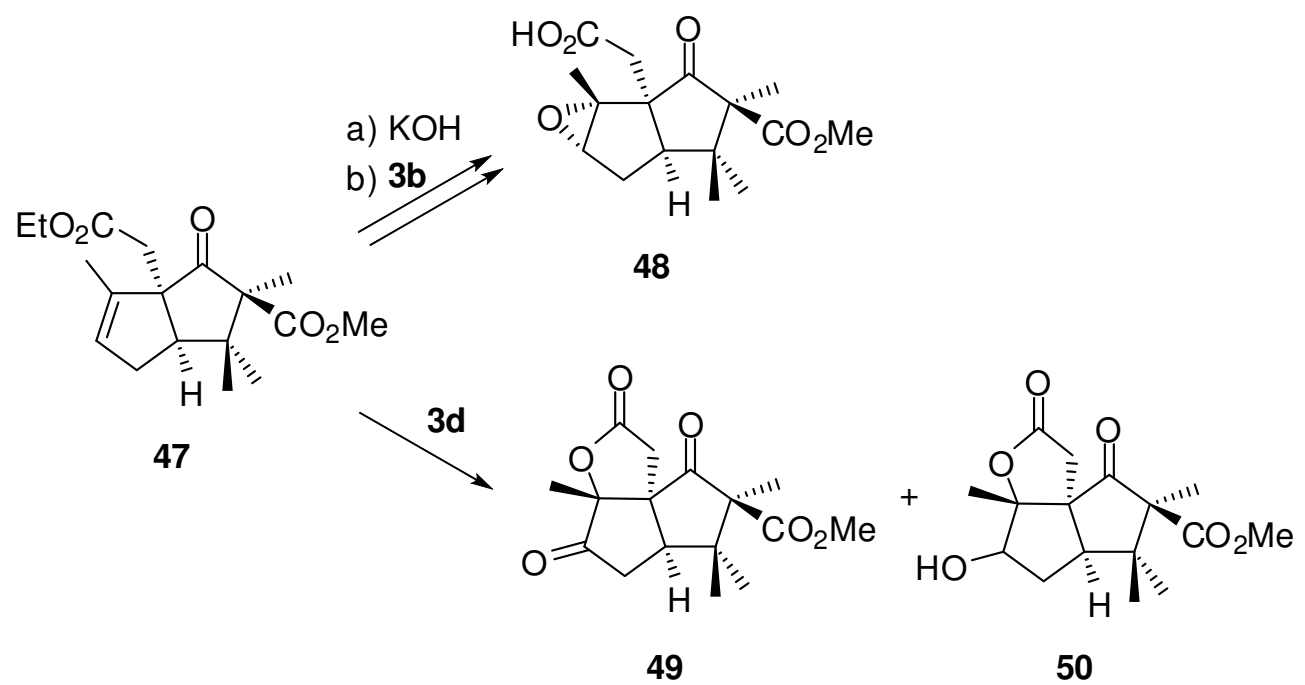

\section{Scheme 19}

The stereochemical outcome of the epoxidation of glycals $\mathbf{5 1}$ and 4-deoxypentanosides $\mathbf{5 2}$ performed with $\mathbf{3 b}$ was reported to depend mainly on the orientation of the OX group at $C(3)$ (Scheme 20). ${ }^{(49)}$ The oxidation in this series led predominantly to the formation of the corresponding trans-oriented epoxide ring to this group, i.e. $\mathbf{5 3}$ and 54, respectively.

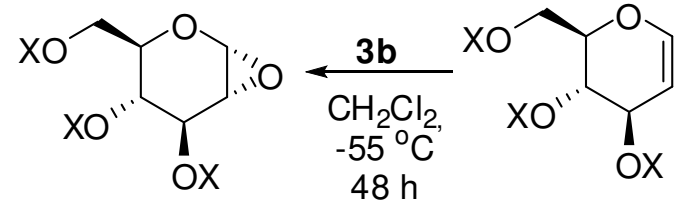

53
51

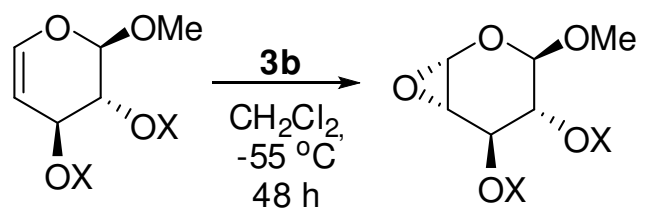

52

54

\section{Scheme 20}

In a related study aimed at the preparation of a carbosugar, the first step of the multistep synthesis comprised the epoxidation of cyclohexene $\mathbf{5 5}$ by treatment with $\mathbf{3 b}$ in acetone solution (Scheme 21). ${ }^{(50)}$ In that case, the formation of a mixture of two stereoisomeric epoxides 56 in a ratio of 2:1 was observed.<smiles>CC(=O)OCC[C@H]1C[C@H](OC(C)=O)C=C[C@H]1OC(C)=O</smiles>

55<smiles>CC(=O)OCC[C@H]1C[C@@H](OC(C)=O)[C@H]2O[C@H]2[C@H]1OC(C)C</smiles>

56 


\section{Scheme 21}

The same research group reported an epoxidation of para-benzoquinone-fused norbornadiene with $\mathbf{3 b}$, and a mixture of exo- and endo-epoxides in a ratio of 3:1 was obtained (Scheme 4). ${ }^{(27)}$

Epoxidation of cyclopentene derivative $\mathbf{5 7}$ by treatment with the in situ generated $\mathbf{3 b}$ led to a $4: 1$ mixture of isomeric epoxides $58 \mathbf{a}$ and $\mathbf{5 8 b}$ (Scheme 22). ${ }^{(51)}$ Whereas the unstable isomer $\mathbf{5 8 a}$ was converted spontaneously into the all-cis-product 59a, the stable isomer $\mathbf{5 8 b}$ was isolated and subsequently transformed to the cis,trans-isomer 59b.

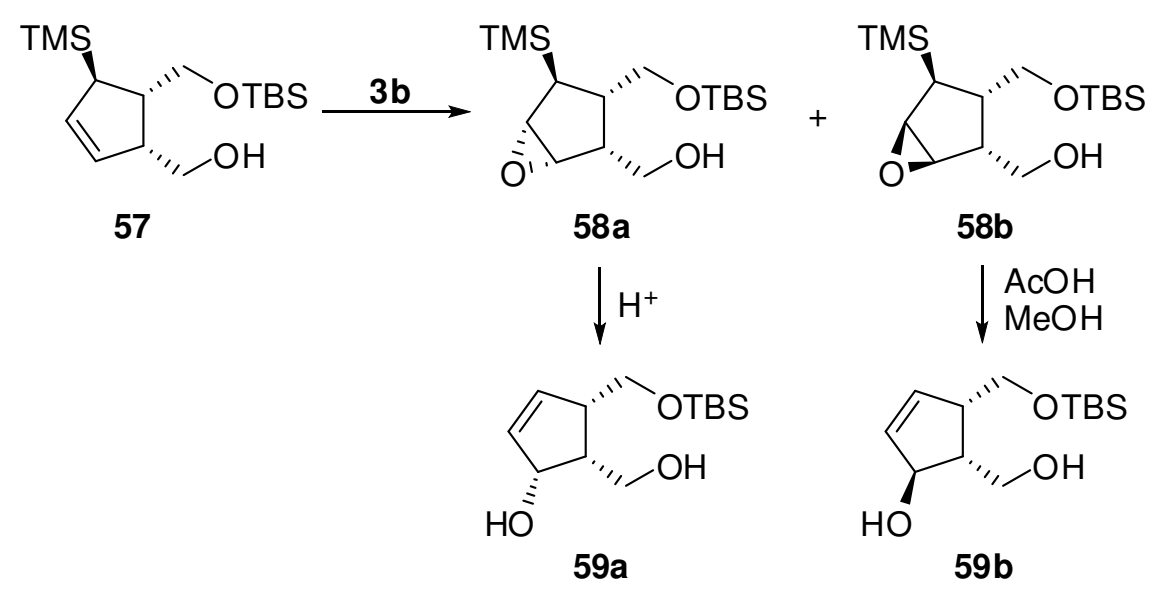

\section{Scheme 22}

Depending on the alkenyl chain, guanine derivatives 60 were transformed into five- or sixmembered N-heterocycles 62 in a one-pot procedure (Scheme 23). ${ }^{(52)}$ The first reaction step of this synthesis was the selective epoxidation of the terminal $\mathrm{C}=\mathrm{C}$ bond, and spontaneous intramolecular nucleophilic attack at the intermediate epoxide 61 results in the formation of the final products. The five-membered imidazolidine derivative with the tertiary $\mathrm{Me}_{2}(\mathrm{OH}) \mathrm{C}$ function was also obtained by this method.

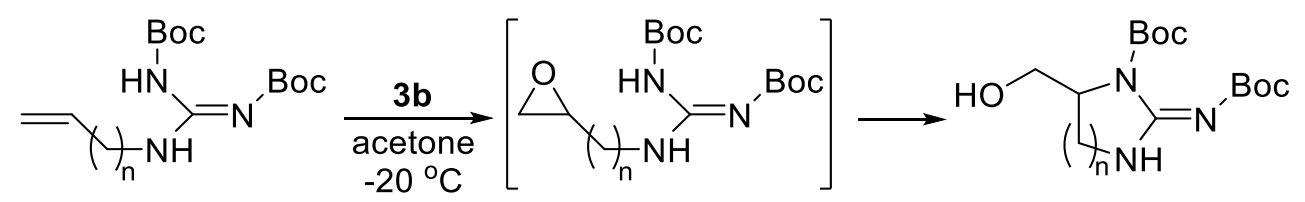

$n=1,2 \quad 60$

$72 \mathrm{~h}$

61

62

\section{Scheme 23}


The gem-difluorinated cyclopentene 63 was efficiently epoxidized by treatment with excess 3d at $-78{ }^{\circ} \mathrm{C}$ to room temperature in trifluoroacetone. The reaction occurred stereoselectively yielding the isomer 64 exclusively (Scheme 24). ${ }^{(53)}$

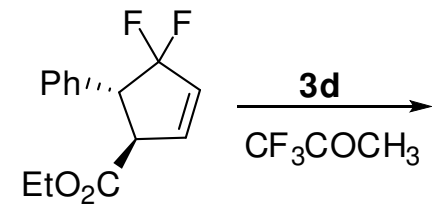

63

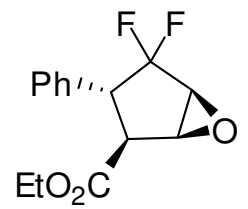

64

\section{Scheme 24}

The conversion of 1-bromonona-1,2-diene (an allene) into a 1:1 (E/Z)-mixture of oct-1-ene 1carboxylic acid was achieved using $\mathbf{3 b}$ in acetone or as the in situ generated reagent in relative low yield. ${ }^{(54)}$ The explanation of the mechanism includes the initial formation of oxiranes, which are converted to the products via a 2-bromocyclopropanone.

Functionalization of single- and multi-walled carbon nanotubes (SWCNT and MWCNT, resp.) is a challenging problem in current organic synthesis. In recent years it was reported that epoxidation of such materials can be efficiently carried out by the involvement of dioxiranes $\mathbf{3}$ as oxidizing reagents. The epoxidation was performed with isolated as well as with in situ generated methyl(trifluoromethyl)dioxirane $(\mathbf{3 d}) .^{(55,56)}$ Both protocols led to epoxidized carbon nanotubes, which subsequently were treated with nucleophiles, e.g. hexane-1,6diamine, yielding the corresponding amino alcohols as a new decoration of the surface. The epoxidation of MWCNTs was also achieved using 2,2,2-trifluoroacetophenone as a precursor of the in situ generated phenyl(trifluoromethyl)dioxirane. ${ }^{(56)}$

A novel epoxidation of SWCNTs filled with metal halides, e.g. Nal and $\mathrm{LuCl}_{3}$, was performed under mild conditions using the isolated $3 \mathbf{d}$ in cold 1,1,1-trifluoropropanone $/ \mathrm{CH}_{2} \mathrm{Cl}_{2}$ solution. ${ }^{(57)}$

Epoxidation with dimethyldioxirane (3b) and subsequent ring opening of the epoxide was applied for the preparation of functionalized metal-organic frameworks. ${ }^{(58)}$ The nucleophilic reagent used for the ring opening was ethanethiol, and the functionalization led to $\beta$ hydroxythioethers 65 (Scheme 25). 


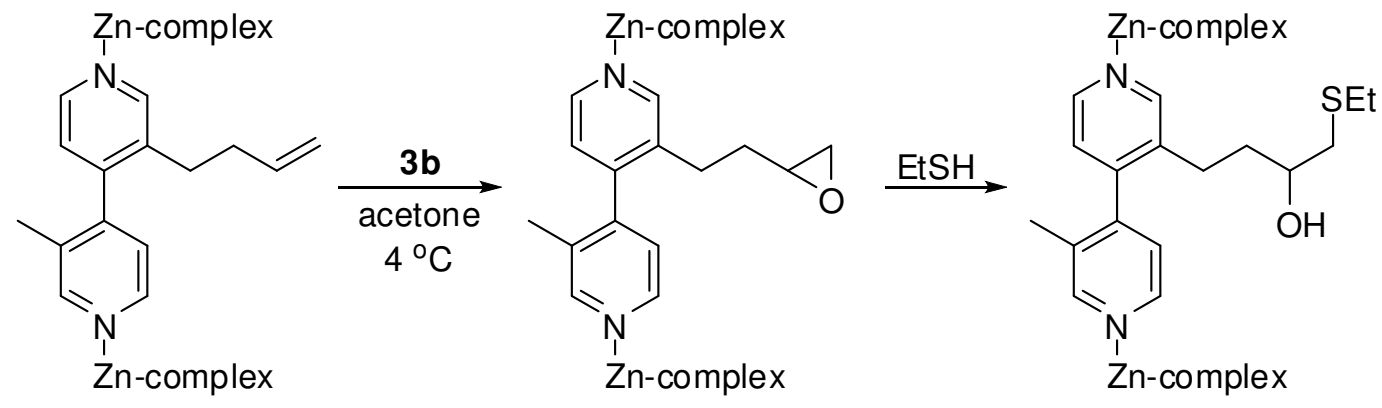

65

\section{Scheme 25}

\subsection{Asymmetric epoxidations}

Diastereoselective epoxidations of enantiopure substrates have been described in the previous chapter. Oxidation of some prochiral alkenes leading to optically active epoxides are also known, and they were performed with in situ generated dioxiranes in the presence of a chiral metal catalyst or a chiral ligand. For example, ethyl (Z)-cinnamate (66) was epoxidized with in situ generated $\mathbf{3 b}$ in the presence of Jacobsen's catalyst and the optically active cis-epoxide $(R, R)-67$ was obtained in high enantioselectivity (ee 78\%) (Scheme 26). ${ }^{(59)}$

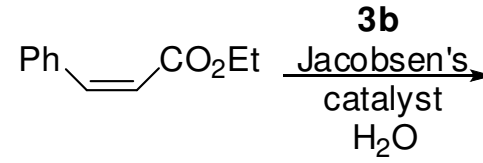

66<smiles>CCOC[C@H]1O[C@H]1P</smiles>

$(R, R)-67$

\section{Scheme 26}

The enantiopure trifluoroacetyl-functionalized dipeptide derivative 68 was applied as precursor of an in situ-generated fluorinated dioxirane. Asymmetric epoxidation of a series of prochiral cyclic and acyclic alkenes with $\mathrm{H}_{2} \mathrm{O}_{2}$ in a 1:1 mixture of tert-amyl alcohol/ $\mathrm{H}_{2} \mathrm{O}$ in the presence of a catalytic amount $(10 \mathrm{~mol} \%)$ of 68 led to the respective oxiranes in good chemical yields and good enantioselectivity. ${ }^{(60)}$ A representative example with 1phenylcyclohexane (69) is depicted in Scheme 27 . The epoxide $\mathbf{7 0}$ was obtained in $88 \%$ yield and the enantiomeric ratio $(e r)$ for the $(S, S)$-isomer was determined to $90.5: 9.5$. 


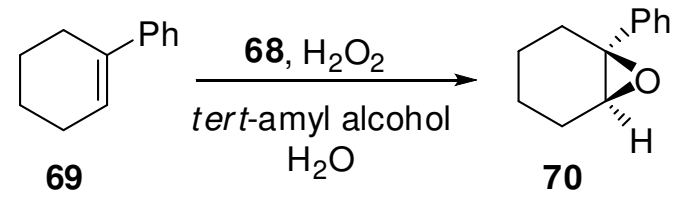

69
$\mathrm{H}_{2} \mathrm{O}$<smiles>CC(C)[C@H](NC(=O)C1CCCN1C(=O)[C@H](C)CC(=O)C(F)(F)F)C(C)N[C@@H](C)c1ccccc1</smiles>

68

\section{Scheme 27}

\subsection{Oxidation of heteroatoms}

Upon treatment of Tröger's bases $\mathbf{7 1}$ with $\mathbf{3 b}$ in acetone solution, the corresponding mono$\mathrm{N}$-oxides $\mathbf{7 2}$ were formed and subsequently converted into the polycyclic hydrazines $\mathbf{7 3}$ after reaction with acetic anhydride (Scheme 28$).{ }^{(61)}$ This sequence of transformations represents a case of demethylenation reaction.<smiles>[R]c1cc2c(c([R])c1[R])CN1CCN2Cc2c1cc([R])c([R])c2[R]</smiles>

71<smiles>[R]c1cc2c(c([R])c1[R])C[N+]1([O-])CCN2Cc2cc([R])c([R])c([R7])c21</smiles>

72

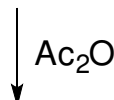<smiles>[R]c1cc2c(c([R])c1[R])CN1Cc3c(cc([R])c([R7])c3C2)N1</smiles>

\section{Scheme 28}

A method for the preparation of polycyclic nitroxyl radicals was elaborated via oxidation of $\mathrm{N}$-heterocycles of type $\mathbf{7 4}$ by treatment with diverse oxidizing reagents. The best results were achieved using $m$-CPBA, but isolated $\mathbf{3 b}$ was also used, and the desired product $\mathbf{7 5}$ was shown to be formed in ca. $10 \%$ yield by EPR spectroscopy (Scheme 29$).{ }^{(62)}$ 


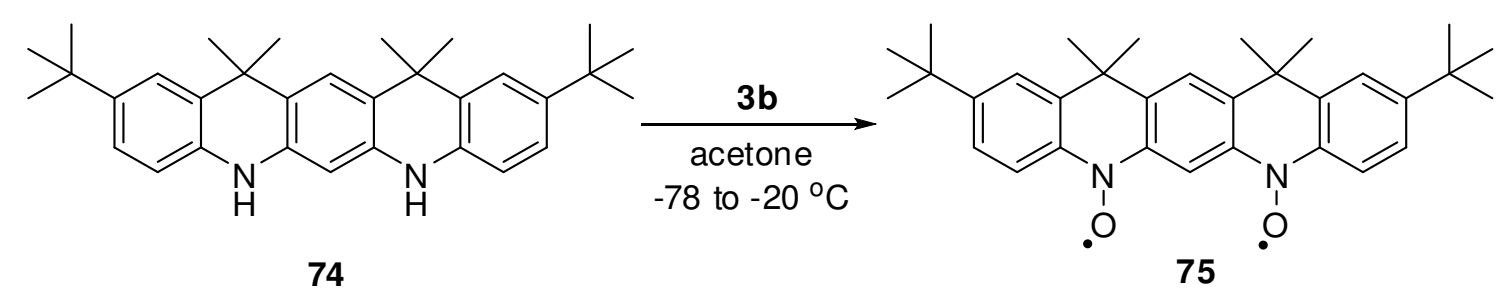

Scheme 29

Oxidations of lactams $\mathbf{7 6}$ with diverse ring size including $\beta$-lactams were efficiently explored for the synthesis of aliphatic $\omega$-nitrocarboxylic acids. ${ }^{(63)}$ In these reactions, the dioxirane $\mathbf{3 d}$ was a perfect oxidizing reagent and the desired products $\mathbf{7 7}$ were obtained as pure materials in nearly quantitative yields (Scheme 30). However, the attempted oxidation of 2,5diketopiperazine under the same conditions was unsuccessful.

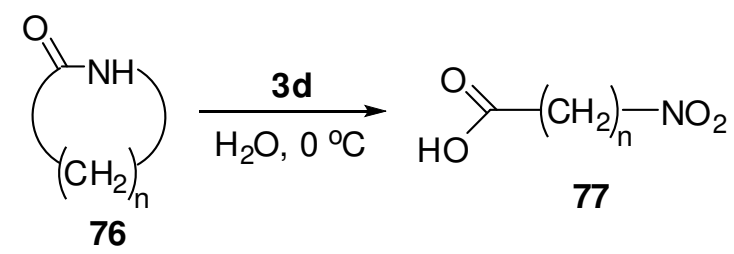

\section{Scheme 30}

The same research group reported on the competitive oxidation processes in reactions of lactams upon treatment with the in situ generated $\mathbf{3 b}$ or $\mathbf{3 d} .^{(64)}$ Depending on the type of the lactam used, $\omega$-nitrocarboxylic acids or monoamides of $\alpha, \omega$-dicarboxylic acids as well as degradation products were obtained.

Reactivities of some guanine-containing fragments of DNA (5mers) and monomeric models were studied upon treatment with oxidizing reagents including $\mathbf{3 b} .^{(65)}$ The observed selectivities of these reactions were the same in the case of using $m$-CPBA as well as of $\mathbf{3 b}$.

The $\alpha$-oxocarboxylic ester derivative $\mathbf{7 9}$ is an important building block for the preparation of kinase inhibitors. ${ }^{(66)} \mathrm{A}$ convenient way for its preparation is the $\mathbf{3 b}$-mediated oxidation of the phosphorus ylide 78 (Scheme 31). The authors did not discuss the mechanism of this reaction but it seems likely that oxidation of the phosphorus atom is an important step of this conversion. 


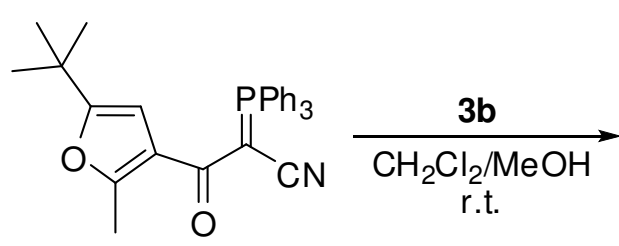

78<smiles>COC(=O)C(=O)c1cc(C(C)(C)C)oc1C</smiles>

$79(92 \%)$

\section{Scheme 31}

Post-synthetic modification of some sulfide-functionalized metal-organic frameworks containing 4,4-biphenyldicarboxylate ligands of type $\mathbf{8 0}$ with $\mathbf{3 b}$ was applied for the oxidation of the sulfur atom leading to the corresponding sulfone $\mathbf{8 1}$ (Scheme 32). ${ }^{(67)}$

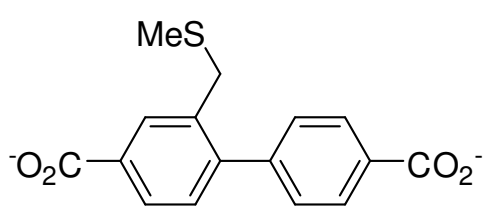

80

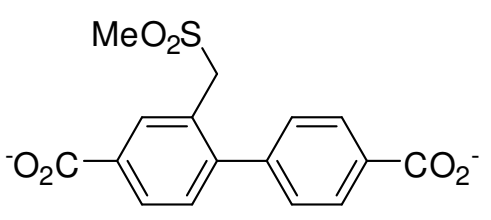

81

\section{Scheme 32}

The S-atoms incorporated in the structure of [Fe-Fe]-hydrogenase models can be oxidized yielding so-called 'sulfur-oxygenated' biomimetics. Along with $m$-CPBA, $\mathbf{3 b}$ was also applied to perform modifications of that type. ${ }^{(68)}$

A series of selenoesters of type $\mathbf{8 2}$ was converted into the corresponding seleninic acids $\mathbf{8 3}$ by treatment with a stoichometric amount of $\mathbf{3 b}$ in moist acetone. ${ }^{(69)}$ A respective example is depicted in Scheme 33. The isolated product $\mathbf{8 3}$ was identified spectroscopically and subsequently smoothly converted into selenonic acid salt 84 in $\mathrm{CH}_{2} \mathrm{Cl}_{2}$ solution using an excess of $\mathbf{3 b}$. 
<smiles>CCOC(CCC[Se]C(=O)Cc1ccccc1)OCCO</smiles>

82<smiles>CCOCC(CC[Se](=O)O)OC(=O)OCC</smiles>

$83(92 \%)$

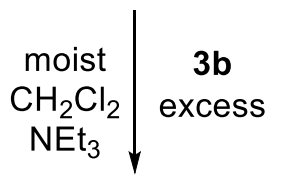

$\underset{\mathrm{Et}_{3} \mathrm{NH}}{+} \underset{\substack{\mathrm{O} \\ \mathrm{O}}}{\stackrel{\mathrm{O}}{\mathrm{O}} e_{84}} \overbrace{(80 \%)}^{\mathrm{OCOC}_{3} \mathrm{H}_{7}} \mathrm{OCOC}_{3} \mathrm{H}_{7}$

$84(80 \%)$

\section{Scheme 33}

Along with functionalized ester substrates of type $\mathbf{8 2}$, pyranose, nucleoside as well as amino acid functionalized derivatives were used in the study.

Oxidation of tosylated $o$-iodoanilines $\mathbf{8 5} \mathbf{a}$-b or 0 -iodophenol $85 \mathrm{c}$ with $\mathbf{3 b}$ in $\mathrm{CH}_{2} \mathrm{Cl}_{2}$ solution at room temperature led after $24 \mathrm{~h}$ to 2 -iodylphenyl derivatives $86 \mathrm{a}-\mathrm{c}$ in high yields (Scheme $34) .{ }^{(70)}$ The product $\mathbf{8 6 a}$ is expected to be a potentially useful hypervalent iodine oxidant.<smiles>[R]c1ccccc1I</smiles>

$85 a-c$<smiles>O=CC1CCCCC1</smiles>
a: $\mathrm{R}=\mathrm{NMeTs}$
b: $\mathrm{R}=\mathrm{NTs}_{2}$
c: $R=O T s$<smiles>[R]c1ccccc1[N+](=O)[O-]</smiles>

86a-c

\section{Scheme 34}

Similarly, oxidation of 3-alkoxy-2-iodopyridines $\mathbf{8 7}$ by using $\mathbf{3 b}$ afforded chemoselectively 3alkoxy-2-iodylpyridines 88 in 92-99\% yield (Scheme 35$).{ }^{(71)}$ 


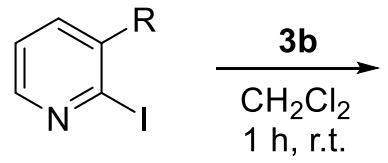

87

$(\mathrm{R}=\mathrm{H}, \mathrm{OMe}, \mathrm{O} \mathrm{Pr}$,

$\mathrm{OPr}, \mathrm{OBu}$ )<smiles>[R]c1cccnc1I(=O)=O</smiles>

88

$(92-99 \%)$

\section{Scheme 35}

lodinated binaphtyls 89 undergo oxidation upon treatment with $\mathbf{3 b}$ in acetone yielding $\lambda^{3}$ iodanes 90 (Scheme 36). ${ }^{(72)}$ The same reaction with some enantiomerically pure starting materials $\mathbf{8 9}$ led to the corresponding pure enantiomers of type $\mathbf{9 0}$ in high yields.<smiles>[R]OC(=O)c1cc2ccccc2c(-c2c([R])c([R])cc3ccccc23)c1I</smiles>

89

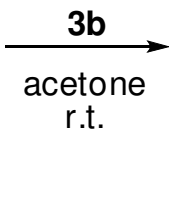

$\mathrm{R}^{1}=\mathrm{Me}$ or $\mathrm{H}$

$\mathrm{R}^{2}=$ OAlk, I or $\mathrm{I}=\mathrm{O}$

$\mathrm{R}^{3}=\mathrm{H}$ or $\mathrm{CO}_{2} \mathrm{R}^{1}$<smiles>[R]c1cc2ccccc2c(-c2c([3H])c(C(=O)O)cc3ccccc23)c1[R]</smiles>

90

\section{Scheme 36}

Whereas the oxidation reaction performed with 89 bearing $R^{2}=$ OAlk and $R^{3}=H$ using 3 equiv. of $\mathbf{3 b}$ afforded the corresponding mono-iodanes $\mathbf{9 0}$, the transformation of diiodo derivatives 89 possessing $R^{2}=1$ and $R^{3}=C_{2} R^{1}$ into diiodanes $90\left(R^{2}=I=O ; R^{3}=C_{2} R^{1}\right)$ required 6 equiv. of $\mathbf{3 b}{ }^{(72)}$ In the same work, selected enantiomerically pure $\lambda^{3}$-iodanes $\mathbf{9 0}$ were explored as oxidizing reagents for asymmetric hydroxylative phenol dearomatization. The tripodal branched trisilanol ligand $\mathbf{9 2}$ was obtained via treatment of trisilane $\mathbf{9 1}$ with $\mathbf{3 b}$. The silane 91 was prepared in a two-step reaction starting with phenyl(trimethoxy)silane (Scheme 37). ${ }^{(73)}$ 


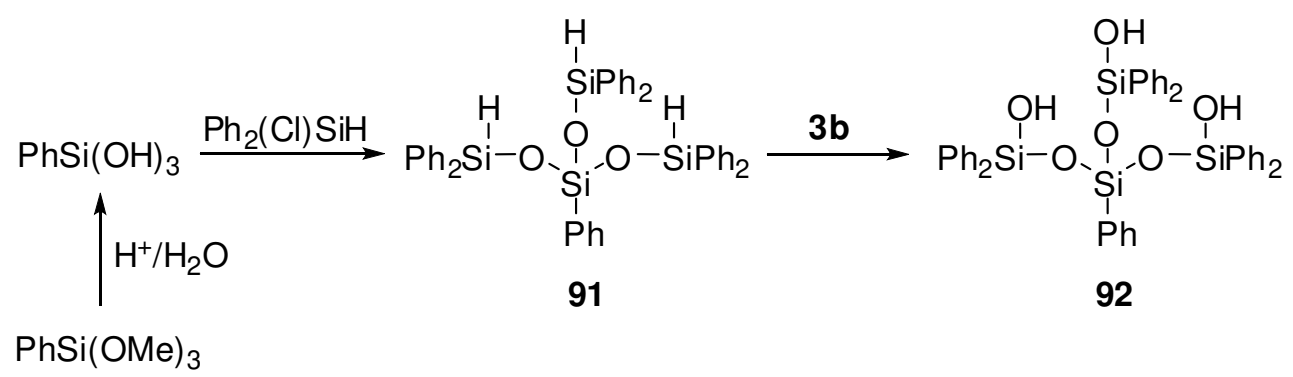

\section{Scheme 37}

\subsection{Oxidation of organometallic compounds}

Dimethyloplatinum(II) complexes with 2,2-bipyridines of type 93 react with $\mathbf{3 b}$ in moist acetone at room temperature immediately to give dihydroxyplatinum(IV) complexes 94 as crystalline products. Under these conditions, the expected oxoplatinum(IV) complex could not be obtained (Scheme 38). ${ }^{(74)}$

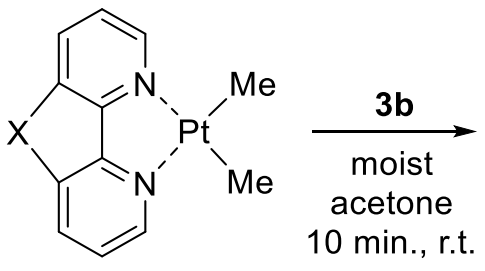

93

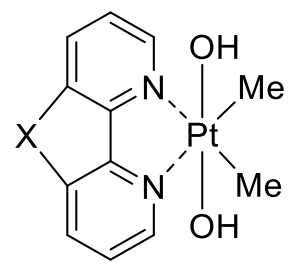

94

$\mathrm{X}=\mathrm{H}, \mathrm{H}$ (no bridge), $\mathrm{NH}, \mathrm{C}=\mathrm{O}$

\section{Scheme 38}

In the case of $93(X=C=0)$, the initially formed dihydroxy derivative underwent spontaneous intramolecular cyclization to give the corresponding polycyclic hemiacetal.

1.14.6 Synthesis of Three-Membered Rings with Two Oxygen and/or Sulfur Atoms

\subsubsection{Dithiiranes}


In the period 2008-2018, no methods for the preparation of isolable amounts of representatives of this class have been reported. Trapping of intermediate dithiiranes by treatment with $\mathrm{Pt}(0)$ complexes or immobilization in low temperature matrices were described in Chapter 1.14.5.1.

\subsubsection{Oxathiiranes}

The only study reported on the parent oxathiirane referred to an Ar-matrix isolated sample at $10 \mathrm{~K}$ (see Chapter 1.14.5.2).

\subsubsection{Dioxiranes}

Recommended new procedures for the preparation of dioxiranes were based on known protocols, comprising oxidations of corresponding ketones, and they were discussed in Chapter 1.14.5.3. A practical and efficient large-scale synthesis of dimethyldioxirane (DMDO, 3b), starting with acetone and Oxone ${ }^{\circledR}$, was published in $2013 .{ }^{(75)}$ In addition, a simplified method for the preparation of $\mathbf{3 b}$, starting with the same substrates, appeared in the same year. ${ }^{(76)}$ In both reports, the concentration of the obtained DMDO solution was assayed by oxidation of an excess of thioanisol following the published procedure. ${ }^{(77)}$

\subsubsection{Important Compounds and Applications}

Until the present time no practical applications of dithiiranes or oxathiiranes were reported. In contrast, some practically useful dioxiranes are described in new patents. For example, oxidation of polyesters and $\mathrm{N}, \mathrm{N}$-dimethylated polyamides obtained by treatment with Oxone $^{\circledR}$ was applied to prepare the dioxirane-containing polymers of type 95 and 96, respectively. ${ }^{(78)}$ These products are recommended as active chemical filters for gas and liquid purification. 


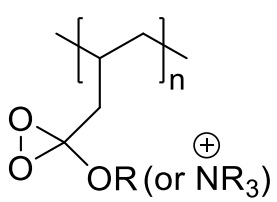

95<smiles></smiles>

96

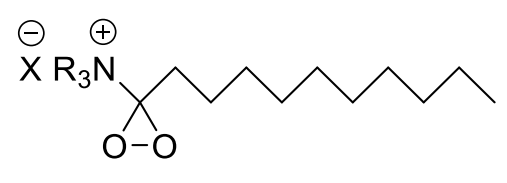

97

Figure 10

A similar procedure was applied for the conversions of diverse long-chain carbonyl compounds, e.g. amides, esters, ketones etc. into corresponding dioxirane derivatives which are useful products for further applications (e.g., oxidants, cleaning, disinfection, contamination agents). ${ }^{(79)}$ A representative formula of quaternary ammonium salts 97 is depicted in Figure 10.

A patented procedure for the preparation of dorzolamide, applied in ophthalmology, comprises chemoselective oxidation of the sulfur atom located in the thiopyrane ring, leading to the corresponding cyclic sulfone. Among diverse oxidation reagents, $\mathbf{3} \mathbf{b}$ (DMDO) is also listed. ${ }^{(80)}$

Dioxiranes produced via oxidation of some ketones with carboxylic peracids were describe in a patent offering a method for manufacturing of new whitening agents. ${ }^{(81)}$

\subsubsection{Conclusions}

In general, dithiiranes constitute a class of three-membered, sulfur-rich heterocycles with limited importance for organic synthesis. However, some representatives were postulated as important intermediates in natural, enzymatically supported processes leading to larger sulfur heterocycles such as 1,2,4-trithiolane, 1,2,4,6-tetrathiepane or 1,2,3,5,6pentathiepane (lenthionine). Dithiiranes are considered as cyclic forms of isomeric thiocarbonyl S-sulfides (thiosulfines) and the existence of equilibria of both compounds is a topic of physico-chemical studies performed preferably in low temperature Ar-matrices. Unstable dithiiranes, generated from respective precursors, can be easily complexed by treatment with $\mathrm{Pt}(0)$-ligands forming so called dithiolato derivatives in which the Pt-atom is inserted into the S-S bond.

Oxathiiranes are only known to a limited extent and they can be considered as cyclic forms of the isomeric thiocarbonyl S-oxides, which are well known and in many instances can be 
isolated and stored at ambient conditions. Some unstable oxathiiranes were postulated as sulfur-transferring reagents in reactions leading to larger sulfur heterocycles. The parent oxathiirane, generated thermally in the gas phase from thioformaldehyde S-oxide, was immobilized and identified in Ar-matrix at $10 \mathrm{~K}$ among other isomeric compounds.

In contrast, dioxiranes constitute a class of three-membered heterocycles with great importance for practical usage in current organic chemistry. In recent years, some dioxiranes found applications as cleaning and disinfection agents. An attractive and well promising field of new exploration of in situ generated dioxiranes relates to clean and selective asymmetric oxidation reactions.

\subsubsection{Further Developments}

The existence of equilibria between dithiiranes and their open-chain isomers is a topic of interest for both coordination chemistry and syntheses of sulfur heterocycles, in which thiocarbonyl S-sulfides behave as 'sulfur-centered' 1,3-dipoles. For that reason, they can react with diverse dipolarophiles yielding the corresponding five-membered cycloadducts. Oxathiiranes can be considered as important intermediates in sulfur/oxygen exchange and this process is of importance for the explanation of desulfurization of some nucleic bases in the presence of active forms of oxygen.

Searching for methods leading to the synthesis of oxathiiranes, isolable and stable at ambient conditions, is a challenging problem of current organic synthesis involving sulfurcontaining substrates.

Dioxiranes will be further used as convenient, clean and highly selective oxidizing reagents. In this context, the chemistry of 'Criegee intermediates' still attracts interest of researchers. Due to potential practical applications, new developments in polymer chemistry, focused on elaboration of methods for manufacturing of dioxiirane containing materials, are also expected.

In addition, elucidation of problems related to structural and electronic properties of threemembered heterocycles with two oxygen and/or sulfur atoms is an attractive topic for theoretical investigations. 


\section{References}

(1) Roje, M.; Portada, T. in Comprehensive Heterocyclic Chemistry III, (C) Elsevier Science, 2008; pp 642-676.

(2) Davis, R. L.; Stiller, J.; Naicker, T.; Jiang, H.; Jørgensen, K. A. Angew. Chem. Int. Ed. 2014, $53,7406-7426$.

(3) Saeed, A.; Larik, F. A.; Lal, B.; Faisal, M.; El-Seedi, H.; Channar, P. A. Synth. Commun. 2017, $47,835-852$.

(4) Adam, W.; Zhao, C.-G.; Saha-Möller, C. R; Jakka, K. Oxidation of Organic Compounds by Dioxiranes, John Wiley \& Sons, Inc., Hoboken, New Yersey, 2009.

(5) Schreiner, P. R.; Reisenauer, H. P.; Romański, J.; Mlostoń, G. J. Am. Chem. Soc. 2010, 132, 7240-7241.

(6) Reisenauer, H. P.; Mlostoń, G.; Romański, J.; Schreiner, P. R. Eur. J. Org. Chem. 2011, 6269-6275.

(7) Mlostoń, G.; Jasiński, M.; Wróblewska, A.; Heimgartner, H. Curr. Org. Chem. 2016, 20, 1359-1369.

(8) Mlostoń, G.; Jasiński, M.; Rygielska, D.; Heimgartner, H. Heterocycles 2011, 83, 765-776.

(9) Bahrami, K.; Khodaei, M. M.; Tajik, M. Synthesis 2010, 4282-4286.

(10) McCaw, P. G.; Buckley, N. M.; Collins, S. G; Maguire, A. R. Eur. J. Org. Chem. 2016, 16301650.

(11) Mlostoń, G.; Romański, J.; Reisenauer, H. P.; Maier, G. Angew. Chem. Int. Ed. 2001, 40, 393-396. 
(12) Murphy, K. V.; Morgan, W. J.; Sun, Z.; Schaefer, H. F. III; Agarwal, J. J. Phys. Chem. A 2017, 121, 998-1005.

(13) Weisheit, T.; Petzold, H.; Görls, H.; Mlostoń, G.; Weigand, W. Eur. J. Inorg. Chem. 2009, 3545-3551.

(14) Mlostoń, G.; Romański, J.; Reisenauer, H. P.; Schreiner, P. R. Phosphorus Sulfur Silicon Relat. Elem. 2011, 186, 1175-1188.

(15) Romański, J.; Reisenauer, H. P.; Petzold, H.; Weigand, W.; Schreiner, P. R.; Mlostoń, G. Eur. J. Org. Chem. 2008, 2998-3003.

(16) Mlostoń, G.; Romański, J.; McKee, M. L.; Reisenauer, H. P.; Schreiner, P. R. Eur. J. Org. Chem. 2010, 2132-2137.

(17) Dumont, E.; Loos, P.-F.; Assfeld, X. Chem. Phys. Lett. 2008, 458, 276-280.

(18) Goyenola, C.; Gueorguiev, G. K.; Stafström, S.; Hultman, L. Chem. Phys. Lett. 2001, 506, 86-91.

(19) Saheb, V. J. Phys. Chem. A 2011, 115, 4263-4269.

(20) Ma, R.; Yuan, D.; Chen, M.; Zhou, M.; Wang, X.; Andrews, L. J. Phys. Chem. A 2009, 113 4976-4981.

(21) Schreiner, P. R.; Reisenauer, H. P.; Romański, J.; Mlostoń, G. Angew. Chem. Int. Ed. 2009, 48, 8133-8136.

(22) Steudel, Y.; Steudel, R.; Wong, M. W. Chem. Eur. J. 2002, 8, 217-228.

(23) Li, J.; Guo, H. J. Phys. Chem. A 2016, 120, 2991-2998. 
(24) Ramakrishnan, R.; Carrington, T., Jr. Spectrochim Acta A: Mol. Biomol. Spectr. 2014, 119, 107-112.

(25) Duarte, D. J. R.; Miranda, M. S.; Esteves da Silva, J. C. G.; Liebman, J. F. Struct. Chem. 2016, 27, 1743-1751.

(26) Düfert, A.; Werz, D. B. J. Org. Chem. 2008, 73, 5514-5519.

(27) Essiz, S.; Dalkilic, E.; Sari, O.; Dastan, A.; Balci, M. Tetrahedron 2017, 73, 1640-1649.

(28) Mc Tush-Camp, D.; Vasquez, P. C.; Baumstark, A. L. Heterocycl. Commun. 2015, 21, 4753.

(29) Bach, R. D. J. Phys. Chem. A 2016, 120, 840-850.

(30) Kuwata, K. T.; Valin, L. C. Chem. Phys. Lett. 2008, 451, 186-191.

(31) Mackenzie-Rae, F. A.; Karton, A.; Saunders, S. M. Phys. Chem. Chem. Phys. 2016, 18, 27991-28002.

(32) Liang, D.; Bian, J.; Deng, L.-W.; Huang, D. J. Funct. Foods 2017, 35, 197-204.

(33) Shahid, S.; Faisal, M.; Saeed, A.; Ghumro, S. A.; El-Seedi, H. R.; Rasheed, S.; Abbas, M.; Larik, F. A.; Channar, P. A.; Fattah, T. A.; Ashraf, Z.; Solangi, Z. A. Curr. Org. Synth. 2018, 15, 1091-1108.

(34) Annese, C.; D’Accolti, L.; Fusco, C.; Gandolfi, R.; Eaton, P. E.; Curci, R. Org. Lett. 2009, 11, 3574-3577.

(35) Fokin, A. A.; Zhuk, T. S.; Pashenko, A. E.; Osipov, V. V.; Gunchenko, P. A.; Serafin, M.; Schreiner, P. R. J. Org. Chem. 2014, 79, 1861-1866. 
(36) Pierce, C. J.; Hilinski, M. K.; Org. Lett. 2014, 16, 6504-6507.

(37) Lesieur, M.; Battilocchio, C.; Labes, R.; Jacq, J.; Genicot, C.; Ley, S. V.; Pasau, P. Chem. Eur. J. 2019, 25, 1203-1207.

(38) Yang, Z.; Yu, P.; Houk, K. N. J. Am. Chem. Soc. 2016, 138, 4237-4242.

(39) Annese, C.; D’Accolti, L.; Dinoi, A.; Fusco, C.; Gandolfi, R.; Curci, R. J. Am Chem. Soc. 2008, 130, 1197-1204.

(40) Kazemnejadi, M.; Shakeri, A.; Nikookar, M.; Shademani, R.; Mohammadi, M. R. Soc. Open Sci. 2018, 5, 171541.

(41) D’Accolti, L.; Annese, C.; Aresta, A.; Fusco, C. J. Heterocycl. Chem. 2014, 51, 1482-1486.

(42) Charbonneau, L.; Foster, X.; Kaliaguine, S. ACS Sustainable Chem. Eng. 2018, 6, $12224-12231$.

(43) Gómez-Fosado, C. G.; Quintero, L.; Fuentes, L.; Sartillo-Piscil, F. Tetrahedron Lett. 2015, $56,5607-5609$.

(44) Kim, H.; Lim, W.; Im, D.; Kim, D.-G.; Rhee, Y. H. Angew. Chem. Int. Ed. 2012, 51, 1205512058.

(45) Mancey, N. C.; Sandon, N.; Auvinet, A.-L.; Butlin, R. J.; Czechtizky, W.; Harrity, J. P. A. Chem. Commun. 2011, 47, 9804-9806.

(46) Jeker, O. F.; Kravina, A. G.; Carreira, E. M. Angew. Chem. Int. Ed. 2013, 52, 12166-12169.

(47) Mizuki, K.; Iwahashi, K.; Murata, N.; Ikeda, M.; Nakai, Y.; Yoneyama, H.; Harusawa, S.; Usami, Y. Org. Lett. 2014, 16, 3760-3763. 
(48) Richers, J.; Pöthig, A.; Herdtweck, E.; Sippel, C.; Hausch, F.; Tiefenbacher, K. Chem. Eur. J. 2017, 23, 3178-3183.

(49) Alberch, L.; Cheng, G.; Seo, S.-K.; Li, X.; Boulineau, F. P.; Wei, A. J. Org. Chem. 2011, 76, 2532-2547.

(50) Kishali, N. H.; Doğan, D.; Sahin, E.; Gunel, A.; Kara, Y.; Balci, M. Tetrahedron 2011, 67, 1193-1200.

(51) Gimazetdinov, A. M.; Almukhametov, A. Z.; Loza, V. V.; Spirikhin, L. V.; Miftakhov, M. S. Mendeleev Commun. 2018, 28, 546-547.

(52) Al Shuhaib, Z.; Arndt, M.; Dennis, M.; Evans, D. M.; Jones, I.; Leitmann V.; Murphy, P. J.; Roberts, D.; Rowles, R.; Sadaghiani, Y. K.; Thornhill, A. J.; Nash, R. J.; Hollinshead, J.; Bartholomew, B.; Tizzard, G. J.; Coles, S. J. Tetrahedron 2017, 73, 845-852.

(53) Orr, D.; Percy, J. M.; Tuttle, T.; Kennedy, A. R.; Harrison, Z. Chem. Eur. J. 2014, 20, 14305-14316.

(54) Braddock, D. C.; Clarke, J.; Rzepa, M. S. Chem. Commun. 2013, 49, 11176-11178.

(55) Annese, C.; D’Accolti, L.; Giambastiani, G.; Mangone, A.; Milella, A.; Tuci, G.; Fusco, C. Eur. J. Org. Chem. 2014, 1666-1671.

(56) Annese, C.; D’Accolti, L.; Armuzza, V.; Da Ros, T.; Fusco, C. Eur. J. Org. Chem. 2015, 3063-3068.

(57) D’Accolti, L.; Gajewska, A.; Kierkowicz, M.; Matincic, M.; Nacci, A.; Sandoval, S.; Ballesteros, B.; Tobias, G.; Da Ros, T.; Fusco, C. Nanomaterials 2018, 8, 137.

(58) Hindelang, K.; Vagin, S. I.; Anger, C.; Rieger, B. Chem. Commun. 2012, 48, 2888-2890. 
(59) Cubillos, J.; Montilla, I.; Montes de Correa, C. Appl. Catal. A 2009, 366, 348-352.

(60) Romney, D. K.; Miller, S. J. Org. Lett. 2012, 14, 1138-1141.

(61) Gao, X.; Hampton, C. S.; Harmata, M. Eur. J. Org. Chem. 2012, 7053-7056.

(62) Rajca, A.; Shiraishi, K.; Boratyński, P. J.; Pink, M.; Miyasaka, M.; Rajca, S. J. Org. Chem. 2011, 76, 8447-8457.

(63) Annese, C.; D’Accolti, L.; Filardi, R.; Tommasi, I.; Fusco, C. Tetrahedron Lett. 2013, 54, 515-517.

(64) Annese, C.; D’Accolti, L.; Fusco, C.; Licini, G.; Zonta, C. Chem. Eur. J. 2017, 23, 259-262.

(65) Ye, W.; Sangaiah, R.; Degen D. E; Gold, A.; Jayaraj, K.; Koshlap, K. M.; Boysen, G.; Williams, J.; Tomer, K. B.; Mocanu, V.: Dicheva, N.; Parker, C. E.; Schaaper, R. M.; Ball, L. M. J. Am. Chem. Soc. 2009, 131, 6114-6123.

(66) Montalban, A. G.; Boman, E.; Chang, C.-D.; Ceide, S. C.; Dahl, R.; Dalesandro, D.; Delaet, N. G. J.; Erb, E.; Gibbs, A.; Kahl, J.; Kessler, L.; Lundström, J.; Miller, S.; Nakanishi, H.; Roberts, E.; Saiah, E.; Sullivan, R.; Wang, Z.; Larson, C. J. Bioorg. Med. Chem. Lett. 2008, 18, 54565459.

(67) Burrows, A. D.; Frost, C. G.; Makon, M. F.; Richardson, C. Chem. Commun. 2009, 42184220.

(68) Liu, T.; Li, B.; Singleton, M. L.; Hall, M. B.; Darensbourg, M. Y. J. Am. Chem. Soc. 2009, $131,8296-8307$.

(69) Abdo, M.; Knapp, S. J. Am. Chem. Soc. 2008, 130, 9234-9235. 
(70) Mailyan, A. K.; Geraskin, I. M.; Nemykin, V. N.; Zhdankin, V. V. J. Org. Chem., 2009, 74, 8444-8447.

(71) Yoshimura, A.; Banek, C. T.; Yusubov, M. S.; Nemykin, V. N; Zhdankin, V. V. J. Org. Chem., 2011, 76, 3812-3819.

(72) Bosset, C.; Coffinier, R.; Peixoto, P. A.; El Assal, M.; Migueu, K.; Sotiropoulos, J.-M.; Pouységu, L.; Quideau, S.; Angew. Chem. Int. Ed. 2014, 53, 9860-9864.

(73) Schax, F.; Braun, B.; Limberg, C. Eur. Inorg. Chem. 2014, 2124-2130.

(74) Pellarin, K. R.; McCready, M. S.; Puddephatt, R. J. Organometallics 2012, 31, 6388-6394.

(75) Mikula, H.; Svatunek, D.; Lumpi, D.; Glöcklhofer, F.; Hametner, C.; Fröhlich, J. Org. Process Res. Dev. 2013, 7, 313-316.

(76) Taber, D. F.; De Matteo, P. W.; Hassan, R. A. Org. Synth. 2013, 90, 350-357.

(77) Adam, W.; Chan, Y. Y.; Cremer, D.; Gauss, J.; Scheutzow, D.; Schindler, M. J. Org. Chem. $1987,52,2800-2803$.

(78) Sjong A.; Anderson, K. K. US Pat. 2013/037178 A1 (Nov 28, 2013).

(79) Anderson, K. US Pat. 2014/187467A1(July 03, 2014).

(80) Kankan, R. N.; Rao, D. R.; Mudgal, S. S. PCT Int. Appl. WO 2008/135770 A2 (Nov 13, 2008).

(81) Boyd, T.; Xu, G.; Adams, R.; Pierce R.; Samaroo, D.; Viscio, D. PCT Int. Appl. WO 2013/95331 A1 (June 27, 2013). 